\title{
Nonlinear Precoding Design for MIMO Amplify-and-Forward Two-Way Relay Systems
}

\author{
Rui Wang, Meixia Tao, Senior Member, IEEE, and Zhengzheng Xiang
}

\begin{abstract}
In traditional multiple-input-multiple-output (MIMO) channel and MIMO one-way relay system, nonlinear precoding design has shown significant performance gain over linear design. In this paper, we aim to study nonlinear precoding design for MIMO amplify-and-forward (AF) two-way relay systems, where nonlinear minimal mean square error (MMSE) decision feedback equalizers (DFEs) are used in two destinations, and linear transmit precoding is applied at the source and relay nodes. We first investigate nonlinear precoding design, where the precoding is only conducted at two sources for a fixed-relay precoder. After some transformations, we prove that this design problem is convex, and an efficient algorithm is provided to find the optimal solution. Then, we consider the nonlinear joint precoding design to further incorporate relay precoding. Due to the nonconvexity of this problem, we first propose an iterative algorithm (Algorithm I) to approach the optimal solution. It is proven that Algorithm $I$ is convergent and can converge to a stationary point of the joint design problem. Moreover, we present a simplified iterative algorithm (Algorithm II) for joint precoding design to reduce the design complexity. It is found that Algorithm II almost achieves the same performance as Algorithm I in most cases. Our simulation results show that the proposed nonlinear joint precoding design significantly outperforms the linear joint precoding design. It is also shown that the choice between the proposed nonlinear source precoding design and the linear relay precoding design is dependent on specific conditions.
\end{abstract}

Index Terms-Minimum mean square error (MMSE), multipleinput-multiple-output (MIMO), nonlinear precoding, nonregenerative relay, two-way relaying.

\section{INTRODUCTION}

$\mathbf{R}$ ECENTLY, two-way relaying has garnered significant interest for its ability in improving the spectral efficiency of wireless transmission in the case of long source-destination distance [1], [2]. As a new cooperative communication scheme, the notion of two-way relaying is to apply the principle of network coding at the relay node to mix the signals received from two sources for subsequent forwarding and then apply, at each destination, self-interference cancelation to extract the desired information. Thus, in contrast to traditional one-way relaying,

Manuscript received November 29, 2011; revised April 2, 2012 and July 2, 2012; accepted July 22, 2012. Date of publication August 3, 2012; date of current version November 6, 2012. This work was supported in part by the National Natural Science Foundation of China under Grant 60902019, by the National 973 Project under Grant 2012CB316100, and by the Program for new Century Excellent Talents in University (NCET) under Grant NCET-11-0331. The review of this paper was coordinated by Prof. W. A. Krzymień.

The authors are with Shanghai Jiao Tong University, Shanghai 200240, China (e-mail: liouxingrui@sjtu.edu.cn; mxtao@sjtu.edu.cn; 7222838@ sjtu.edu.cn).

Color versions of one or more of the figures in this paper are available online at http://ieeexplore.iee.org.

Digital Object Identifier 10.1109/TVT.2012.2211631 half of channel usages can be saved to complete one round of information exchange between two sources. On the other hand, the multiple-input-multiple-output (MIMO) technique has been considered to be a significant technical breakthrough in wireless communications. Similar to conventional point-topoint and one-way relay systems, incorporating the MIMO technique into a two-way relay system is expected to further enhance throughput and achieve more reliable transmissions.

To fully realize the benefits of MIMO and two-way relaying, conducting precoding at both the source nodes and the relay node by making use of channel state information (CSI) is crucial. A few studies have investigated the precoding design for MIMO two-way relay systems. For example, in [3]-[5], the relay precoding design for a MIMO two-way relay system where multiple antennas are only equipped at the relay node is considered. Then, in [6]-[9], a more complex case is studied, where both the source and relay nodes are equipped with multiple antennas. Under the decode-and-forward (DF) relay strategy, [6] investigates the relay precoding design and compares the capacity gain for two different reencoding operations. Different from [6], in [7]-[9], the amplify-and-forward (AF) relay strategy is considered. In addition to relay precoding, [7][9] also incorporate source precoding such that source and relay precoding are jointly designed to improve system performance. Other than traditional two-user networks, the precoding of MIMO two-way relaying has also been extended to multiuser networks [10]-[12].

It is worth noting that the aforementioned precoding designs for MIMO two-way relay systems are linear. Although the nonlinear precoding design has been well studied in MIMO point-to-point systems [13], [14] and MIMO one-way relay systems [15], [16], to the best of our knowledge, it has not been considered in MIMO two-way relay systems. Since nonlinear precoding has been shown to have better performance than linear precoding [13]-[16], incorporating nonlinear precoding into MIMO two-way relay systems is expected to further improve system performance. Thus, in this paper, we aim to investigate the nonlinear precoding design for MIMO two-way relay systems. Specifically, we adopt the AF relay strategy for its simplicity of implementation. For the considered nonlinear precoding scheme, we assume that linear precodings are exploited at the source and relay nodes, and nonlinear decision feedback equalizers (DFEs) are applied at two destinations. Our objective is to minimize the total mean square error (TotalMSE) of two destinations. Thus, we refer to such receiver as minimum mean square error (MMSE)-DFE receiver, as in [15].

First, we study nonlinear precoding design at two sources for a fixed-relay precoder. After using some transformations, 


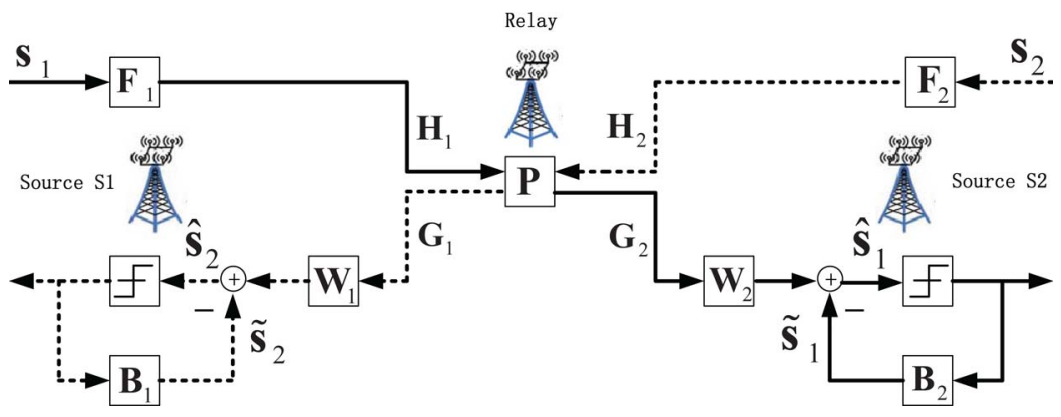

Fig. 1. Nonlinear precoding MIMO two-way relay system.

we prove that this design problem is convex, and an efficient algorithm is provided to find the optimal solution. Then, we study the more complex nonlinear joint precoding design at both the source nodes and the relay node. It is shown that this joint precoding design problem is nonconvex. To make the design problem tractable, we first propose an iterative algorithm (Algorithm I) to approach the optimal solution based on alternating optimization. We prove that Algorithm I is convergent and can converge to a stationary point of the joint design problem. Based on the insights obtained from Algorithm I, we further propose a simplified version of the iterative algorithm (Algorithm II) by relaxing the relay power constraint when optimizing the source precoders during each iteration. It is found that the simplified method, i.e., Algorithm II, almost achieves the same performance as Algorithm I in most cases. Our simulation results show that the proposed nonlinear joint precoding design can significantly outperform the linear joint precoding design. However, the choice between the proposed nonlinear source precoding design and the linear relay precoding design is dependent on the conditions.

Notations: $\mathcal{E}(\cdot)$ denotes the expectation over the random variables within the bracket. $\otimes$ denotes the Kronecker operator. $\operatorname{vec}(\cdot)$ and $\operatorname{mat}(\cdot)$ signify the matrix vectorization operator and the corresponding inverse operation, respectively. $\operatorname{Tr}(\mathbf{A}),|\mathbf{A}|$, and $\mathbf{A}^{-1}$ stand for the trace, determinant, and inverse of matrix A, respectively. Superscripts $(\cdot)^{T},(\cdot)^{*}$, and $(\cdot)^{H}$ denote transpose, conjugate, and conjugate transpose, respectively. $\mathbb{C}^{x \times y}$ denotes the space of $x \times y$ matrices with complex entries. $\mathbb{S}^{N}$ and $\mathbb{S}_{+}^{N}$ denote the set of symmetric $N \times N$ matrices and the set of positive semidefinite $N \times N$ matrices, respectively. The distribution of a circular symmetric complex Gaussian vector with mean vector $\mathrm{x}$ and covariance matrix $\boldsymbol{\Sigma}$ is denoted by $\mathcal{C N}(\mathbf{x}, \mathbf{\Sigma})$.

\section{SySTEM Model}

Consider an $(N, M, N)$ MIMO AF two-way relay system where two $N$-antenna source nodes denoted as $S_{1}$ and $S_{2}$ want to exchange messages through an $M$-antenna relay node denoted as $R$. Here, we assume that $N$ data streams are transmitted from each source to fully utilize the multiplexing gain. To efficiently transmit $N$ data streams, we assume that $M \geq N$ as in [7] and [8]. The information exchange takes two time slots, as shown in Fig. 1. In the first time slot (also referred to as multiple access control (MAC) phase), two source nodes $S_{1}$ and $S_{2}$ simultaneously transmit their signals to relay node
$R$. Thus, the received $M \times 1$ signal vector at the relay node is expressed as

$$
\mathbf{y}_{R}=\mathbf{H}_{1} \mathbf{F}_{1} \mathbf{s}_{1}+\mathbf{H}_{2} \mathbf{F}_{2} \mathbf{s}_{2}+\mathbf{n}_{R}
$$

where $\mathbf{H}_{i} \in \mathbb{C}^{M \times N}$, for $i=1,2$, is the full-rank channel matrix from $S_{i}$ to $R, \mathbf{F}_{i} \in \mathbb{C}^{N \times N}$ denotes the transmit precoding matrix at $S_{i}, \mathbf{s}_{i}$ represents the transmit signal from $S_{i}$, and $\mathbf{n}_{R}$ denotes the additive noise vector at $R$, with $\mathbf{n}_{R} \sim \mathcal{C N}\left(\mathbf{0}, \sigma_{R}^{2} \mathbf{I}_{M}\right)$. Here, we assume that $\mathbf{s}_{i}$ is normalized as $\mathcal{E}\left(\mathbf{s}_{i} \mathbf{s}_{i}^{H}\right)=\mathbf{I}_{N}$, and the power constraint at two sources can thus be denoted as

$$
\operatorname{Tr}\left(\mathbf{F}_{i} \mathbf{F}_{i}^{H}\right) \leq P_{i}, \quad i=1,2
$$

where $P_{i}$ is the maximum transmit power at $S_{i}$.

Upon receiving $\mathbf{y}_{R}$, the relay node amplifies it by multiplying it with a precoding matrix $\mathbf{P} \in \mathbb{C}^{M \times M}$. Therefore, the $M \times 1$ transmit signal vector from the relay node can be expressed as $\mathbf{x}_{R}=\mathbf{P y}_{R}$. We assume that the maximum power at the relay node is $P_{R}$, which yields

$$
\operatorname{Tr}\left\{\mathbf{P}\left(\mathbf{H}_{1} \mathbf{F}_{1} \mathbf{F}_{1}^{H} \mathbf{H}_{1}^{H}+\mathbf{H}_{2} \mathbf{F}_{2} \mathbf{F}_{2}^{H} \mathbf{H}_{2}^{H}+\sigma_{R}^{2} \mathbf{I}_{M}\right) \mathbf{P}^{H}\right\} \leq P_{R} .
$$

Then, the received signal at $S_{i}$ after the second time slot [which is also referred to as the broadcast (BC) phase] can be written as

$$
\mathbf{y}_{i}=\mathbf{G}_{i} \mathbf{P} \mathbf{H}_{\bar{i}} \mathbf{F}_{\bar{i}} \mathbf{s}_{\bar{i}}+\mathbf{G}_{i} \mathbf{P} \mathbf{H}_{i} \mathbf{F}_{i} \mathbf{s}_{i}+\mathbf{G}_{i} \mathbf{P n}_{R}+\mathbf{n}_{i}, \quad i=1,2
$$

where $\bar{i}=2$ if $i=1$ and $\bar{i}=1$ if $i=2, \mathbf{G}_{i} \in \mathbb{C}^{N \times M}$ is the fullrank channel matrix from $R$ to $S_{i}$, and $\mathbf{n}_{i}$ denotes the additive noise vector at $S_{i}$, with $\mathbf{n}_{i} \sim \mathcal{C N}\left(\mathbf{0}, \sigma_{i}^{2} \mathbf{I}_{N}\right)$. Subtracting the backpropagated self-interference term $\mathbf{G}_{i} \mathbf{P} \mathbf{H}_{i} \mathbf{F}_{i} \mathbf{s}_{i}$ from (3) yields the equivalent received signal vector at each destination node as

$$
\mathbf{y}_{i}=\mathbf{D}_{i} \mathbf{s}_{\bar{i}}+\mathbf{G}_{i} \mathbf{P n}_{R}+\mathbf{n}_{i}, \quad i=1,2
$$

where $\mathbf{D}_{i}=\mathbf{G}_{i} \mathbf{P H}_{\bar{i}} \mathbf{F}_{\bar{i}}$ is the equivalent end-to-end MIMO channel matrix for $S_{i}$.

At each destination $S_{i}$, the MMSE-DFE receiver is implemented by using a feedforward matrix filter $\mathbf{W}_{i} \in \mathbb{C}^{N \times N}$ and a feedback matrix filter $\mathbf{B}_{i} \in \mathbb{C}^{N \times N}$, as shown in Fig. 1. Note that $\mathbf{B}_{i}$ is a strictly upper triangular matrix, which implies that the detection of transmit symbols starts from the $N$ th row and the detection of the $k$ th symbol is precoded by subtracting the effect of previously decoded symbols. Assuming correct 
TABLE I

ILLUSTRATION OF SIGNALING OVERHEAD

\begin{tabular}{l|c|c|c|c}
\hline \hline & & \multicolumn{2}{c}{ Non-reciprocal } \\
\cline { 2 - 5 } & CSI & Precoding & CSI & Precoding \\
\hline & & & $S_{1} \rightarrow R: \mathbf{G}_{1}$ & \\
scheme A) & $R \rightarrow S_{1}: \mathbf{H}_{2}$ & $R \rightarrow S_{1}: \mathbf{P}, \mathbf{F}_{1}, \mathbf{F}_{2}, \mathbf{B}_{1}, \mathbf{W}_{1}$ & $S_{2} \rightarrow R: \mathbf{G}_{2}$ & $R \rightarrow S_{1}: \mathbf{P}, \mathbf{F}_{1}, \mathbf{F}_{2}, \mathbf{B}_{1}, \mathbf{W}_{1}$ \\
& $R \rightarrow S_{2}: \mathbf{H}_{1}$ & $R \rightarrow S_{2}: \mathbf{P}, \mathbf{F}_{1}, \mathbf{F}_{2}, \mathbf{B}_{2}, \mathbf{W}_{2}$ & $R \rightarrow S_{1}: \mathbf{H}_{1}, \mathbf{H}_{2}$ & $R \rightarrow S_{2}: \mathbf{P}, \mathbf{F}_{1}, \mathbf{F}_{2}, \mathbf{B}_{2}, \mathbf{W}_{2}$ \\
& & $R \rightarrow S_{2}: \mathbf{H}_{1}, \mathbf{H}_{2}$ & \\
\hline & & $S_{1} \rightarrow R: \mathbf{G}_{1}$ & \\
scheme B) & \multirow{2}{*}{ No } & $S_{2} \rightarrow R: \mathbf{G}_{2}$ & No \\
& $R \rightarrow S_{1}: \mathbf{H}_{2}$ & & $R \rightarrow S_{1}: \mathbf{H}_{1}, \mathbf{H}_{2}, \mathbf{G}_{2}$ & \\
& $R \rightarrow S_{2}: \mathbf{H}_{1}$ & & $R \rightarrow S_{2}: \mathbf{H}_{1}, \mathbf{H}_{2}, \mathbf{G}_{1}$ & \\
\hline \hline
\end{tabular}

previous decisions as in [13]-[16], the input to the detector $\hat{\mathbf{s}}_{i}$ can be written as

$$
\begin{aligned}
\hat{\mathbf{s}}_{\bar{i}} & =\mathbf{W}_{i} \mathbf{y}_{i}-\mathbf{B}_{i} \mathbf{s}_{\bar{i}} \\
& =\left(\mathbf{W}_{i} \mathbf{D}_{i}-\mathbf{B}_{i}\right) \mathbf{s}_{\bar{i}}+\mathbf{W}_{i} \mathbf{G}_{i} \mathbf{P n}_{R}+\mathbf{W}_{i} \mathbf{n}_{i} .
\end{aligned}
$$

Then, the error vector between $\hat{\mathbf{s}}_{\bar{i}}$ and $\mathbf{s}_{\bar{i}}$ is given by

$$
\begin{aligned}
\mathbf{e}_{\bar{i}} & =\hat{\mathbf{s}}_{\bar{i}}-\mathbf{s}_{\bar{i}} \\
& =\left(\mathbf{W}_{i} \mathbf{D}_{i}-\mathbf{I}_{N}-\mathbf{B}_{i}\right) \mathbf{s}_{\bar{i}}+\mathbf{W}_{i} \mathbf{G}_{i} \mathbf{P} \mathbf{n}_{R}+\mathbf{W}_{i} \mathbf{n}_{i} .
\end{aligned}
$$

The sum MSE of detector input is thereby derived as

$$
\begin{aligned}
E_{\bar{i}}=\mathcal{E}\left(\mathbf{e}_{i}^{H} \mathbf{e}_{\bar{i}}\right) & =\operatorname{Tr}\left\{\left(\mathbf{W}_{i} \mathbf{D}_{i}-\mathbf{U}_{i}\right)\left(\mathbf{W}_{i} \mathbf{D}_{i}-\mathbf{U}_{i}\right)^{H}\right. \\
& \left.+\mathbf{W}_{i}\left(\sigma_{R}^{2} \mathbf{G}_{i} \mathbf{P} \mathbf{P}^{H} \mathbf{G}_{i}^{H}+\sigma_{i}^{2} \mathbf{I}_{N}\right) \mathbf{W}_{i}^{H}\right\}
\end{aligned}
$$

where $\mathbf{U}_{i}=\mathbf{I}_{N}+\mathbf{B}_{i}$, for $i=1,2$.

Before leaving this section, we provide some discussions on the synchronization issue and the signaling overhead for obtaining the CSI and the precoding information in the system. In this paper, we assume perfect synchronization at the MAC phase, so that the transmissions of the two source nodes align. In addition, we assume that the channel characteristics of each link change slowly enough so that they can be perfectly estimated at each receiver by using pilot symbols or training sequences. The required signaling overhead depends on the node in the system conducting the joint precoding design. In the following, we discuss two specific scenarios.

1) The relay node performs joint precoding design and then broadcasts the resulting precoding and decoding matrices to the source nodes. ${ }^{1}$

2) Each node simultaneously performs the joint design.

The illustration of overall signaling overhead is presented in Table I, where we suppose that the corresponding channel matrix is estimated at each destination node. For completeness, both reciprocal channels, i.e., $\mathbf{G}_{i}=\mathbf{H}_{i}^{T}$, and nonreciprocal channels, where $\mathbf{G}_{i}$ is independent with $\mathbf{H}_{i}$, are considered, respectively. From Table I, it is easy to find that each scheme has its own merits. Specifically, the first one can lower the computational complexity at two source nodes, whereas it requires much signaling overhead to feed back the precoding

\footnotetext{
${ }^{1}$ The joint precoding can also be designed at one of the source nodes, but it requires more signaling overhead to acquire CSI and precoding information than performing the joint design at the relay node.
}

information. The second one needs less signaling overhead; however, it requires that all the nodes have enough processing ability to conduct the precoding design.

\section{Nonlinear Precoding Designs}

Our goal is to minimize the Total-MSE of two users. Thus, the precoding design optimization is formulated as

$$
\min _{\mathbf{F}_{i}, \mathbf{P}, \mathbf{W}_{i}, \mathbf{U}_{i}, i=1,2} E_{1}+E_{2}
$$$$
\text { s.t. (1), (2). }
$$

From (8), it is not hard to see that the feedforward matrix $\mathbf{W}_{i}$, for $i=1,2$, is irrelevant to the power constraint. Since the objective function in (8) is convex with respect to $\mathbf{W}_{i}$, the optimal $\mathbf{W}_{i}$ can be obtained by equating the gradient of the objective function in (8) to zero, which is derived as

$$
\mathbf{W}_{i}=\mathbf{U}_{i} \mathbf{D}_{i}^{H}\left(\mathbf{D}_{i} \mathbf{D}_{i}^{H}+\mathbf{R}_{i}\right)^{-1}, \quad i=1,2
$$

where $\mathbf{R}_{i}=\sigma_{R}^{2} \mathbf{G}_{i} \mathbf{P P}^{H} \mathbf{G}_{i}^{H}+\sigma_{i}^{2} \mathbf{I}_{N}$. Substituting the optimal $\mathbf{W}_{i}$, for $i=1,2$, into (7), we get an alternative expression of MSE as

$$
E_{\bar{i}}=\operatorname{Tr}\left\{\mathbf{U}_{i}\left(\mathbf{I}_{N}+\mathbf{D}_{i}^{H} \mathbf{R}_{i}^{-1} \mathbf{D}_{i}\right)^{-1} \mathbf{U}_{i}^{H}\right\}, \quad i=1,2 .
$$

Then, the nonlinear precoding design problem can be equivalently rewritten as

$$
\begin{aligned}
& \underset{\mathbf{F} i, \mathbf{P}, \mathbf{U}_{i}, i=1,2}{\sum_{i=1}^{2}} \operatorname{Tr}\left\{\mathbf{U}_{i}\left(\mathbf{I}_{N}+\mathbf{D}_{i}^{H} \mathbf{R}_{i}^{-1} \mathbf{D}_{i}\right)^{-1} \mathbf{U}_{i}^{H}\right\} \\
& \text { s.t. (1), (2). }
\end{aligned}
$$

We can verify that (11) is nonconvex and that the optimal solution is not easily tractable. It is worth noting that, from a practical application point of view, operating the joint nonlinear precoding at both the source and relay nodes may not be feasible in some scenarios. Thus, in the following, we first consider a simple case where the precoding is only conducted at two source nodes for a fixed-relay precoder. After that, we consider a more complex case where the precoding is exploited at both the source and relay nodes. 


\section{A. Nonlinear Source Precoding Design for a Fixed-Relay Precoder}

This precoding scheme is suitable to a scenario where the relay node does not have enough processing ability to enable the joint precoding design and the system signaling overhead is strictly limited. Thus, applying a fixed-relay precoder can lower the computational complexity at the relay node and, on the other hand, can reduce the signaling overhead if the precoding is designed at two source nodes. The corresponding precoding design problem is thereby yielded as

$$
\begin{aligned}
& \min _{\mathbf{F}_{i}, \mathbf{U}_{i}, i=1,2} \sum_{i=1}^{2} \operatorname{Tr}\left\{\mathbf{U}_{i}\left(\mathbf{I}_{N}+\mathbf{D}_{i}^{H} \mathbf{R}_{i}^{-1} \mathbf{D}_{i}\right)^{-1} \mathbf{U}_{i}^{H}\right\} \\
& \text { s.t. (1), (2). }
\end{aligned}
$$

Although (12) exhibits a simpler form than (11), it is still a nonconvex problem. To help derive the optimal solution of (12), we resort to minimizing a lower bound of (10) as in [13], which is denoted as

$$
E_{\bar{i}}^{\mathrm{lb}}=N\left|\mathbf{I}_{N}+\mathbf{D}_{i}^{H} \mathbf{R}_{i}^{-1} \mathbf{D}_{i}\right|^{-1 / N}, \quad i=1,2 .
$$

To obtain the lower bound (13) from (10), we have used the arithmetic-geometric mean inequality $\operatorname{Tr}(\mathbf{X}) \geq M|\mathbf{X}|^{1 / M}$, with $\mathbf{X}$ being an $M \times M$ positive semidefinite matrix. At the end of this section, we shall show that suitable $\mathbf{F}_{i}$ and $\mathbf{B}_{i}$ (i.e., $\mathbf{U}_{i}$ ) can be chosen to achieve this lower bound. According to (13), the optimization problem to solve $\mathbf{F}_{i}$ is described as ${ }^{2}$

$$
\begin{aligned}
\min _{\mathbf{F}_{1}^{\mathrm{lb}}, \mathbf{F}_{2}^{\mathrm{lb}}} & \sum_{i=1}^{2}\left|\mathbf{I}_{N}+\mathbf{F}_{\bar{i}}^{\mathrm{lb}}{ }^{H} \mathbf{H}_{i}^{H} \mathbf{P}^{H} \mathbf{G}_{i}^{H} \mathbf{R}_{i}^{-1} \mathbf{G}_{i} \mathbf{P} \mathbf{H}_{i} \mathbf{F}_{\bar{i}}^{\mathrm{lb}}\right|^{-\frac{1}{N}} \\
\text { s.t. } & \operatorname{Tr}\left(\mathbf{F}_{i}^{\mathrm{lb}} \mathbf{F}_{i}^{\mathrm{lb}}{ }^{H}\right) \leq P_{i}, \quad i=1,2 \\
& \sum_{i=1}^{2} \operatorname{Tr}\left(\mathbf{F}_{i}^{\mathrm{lb}}{ }^{H} \mathbf{H}_{i}^{H} \mathbf{P}^{H} \mathbf{P} \mathbf{H}_{i} \mathbf{F}_{i}^{\mathrm{lb}}\right) \leq P_{R}^{\prime}
\end{aligned}
$$

where $P_{R}^{\prime}=P_{R}-\sigma_{R}^{2} \operatorname{Tr}\left\{\mathbf{P} \mathbf{P}^{H}\right\}$. Since (14) is still nonconvex with respect to $\mathbf{F}_{i}^{\mathrm{lb}}$, to derive the optimal solution, we rewrite (14) as follows by introducing $\mathbf{Q}_{i}=\mathbf{F}_{i}^{\mathrm{lb}} \mathbf{F}_{i}^{\mathrm{lb}}{ }^{H}$ :

$$
\begin{aligned}
\min _{\mathbf{Q}_{1}, \mathbf{Q}_{2} \succeq 0} & \left|\mathbf{I}_{N}+\mathbf{M}_{1} \mathbf{Q}_{1}\right|^{-1 / N}+\left|\mathbf{I}_{N}+\mathbf{M}_{2} \mathbf{Q}_{2}\right|^{-\frac{1}{N}} \\
\text { s.t. } & \operatorname{Tr}\left(\mathbf{Q}_{i}\right) \leq P_{i}, \quad i=1,2 \\
& \operatorname{Tr}\left\{\mathbf{N}_{1} \mathbf{Q}_{1}+\mathbf{N}_{2} \mathbf{Q}_{2}\right\} \leq P_{R}^{\prime}
\end{aligned}
$$

where $\mathbf{M}_{\bar{i}}=\mathbf{H}_{\bar{i}}^{H} \mathbf{P}^{H} \mathbf{G}_{i}^{H} \mathbf{R}_{i}^{-1} \mathbf{G}_{i} \mathbf{P} \mathbf{H}_{\bar{i}}$, and $\mathbf{N}_{i}=\mathbf{H}_{i}^{H} \mathbf{P}^{H} \mathbf{P} \mathbf{H}_{i}$, for $i=1,2$. To proceed in solving (15), we have the following lemma, the proof of which is given in Appendix A:

Lemma 1: The optimization problem (15) is convex with respect to $\mathbf{Q}_{1}$ and $\mathbf{Q}_{2}$.

Although (15) is a convex optimization problem, it is not easy to obtain a closed-form solution. To gain more insight

\footnotetext{
${ }^{2}$ For notation convenience, we denote the designed source precoders from the lower bound as $\mathbf{F}_{i}^{\mathrm{lb}}, i=1,2$.
}

into problem (15), we derive the optimal structure of $\mathbf{Q}_{i}$, for $i=1,2$, in the following lemma:

Lemma 2: The optimal solution of $\mathbf{Q}_{i}$, for $i=1,2$, in (15) has the form

$$
\mathbf{Q}_{i}=\mathbf{M}_{i}^{-\frac{1}{2}} \tilde{\mathbf{U}}_{i} \boldsymbol{\Sigma}_{Q i} \tilde{\mathbf{U}}_{i}^{H} \mathbf{M}_{i}^{-\frac{1}{2}}, \quad i=1,2
$$

where $\Sigma_{Q i}$ is a diagonal matrix, with its entries arranged in increasing order; and $\tilde{\mathbf{U}}_{i}$ is the eigenvector matrix of $\mathbf{M}_{i}^{-1 / 2} \mathbf{Z}_{i} \mathbf{M}_{i}^{-1 / 2}$, where $\mathbf{Z}_{1}=\lambda_{1} \mathbf{I}_{N}+\lambda_{3} \mathbf{N}_{1}$ and $\mathbf{Z}_{2}=$ $\lambda_{2} \mathbf{I}_{N}+\lambda_{3} \mathbf{N}_{2}$, with $\lambda_{1}, \lambda_{2}$, and $\lambda_{3}$ being three nonnegative scalars. The eigenvalue decomposition of $\mathbf{M}_{i}^{-1 / 2} \mathbf{Z}_{i} \mathbf{M}_{i}^{-1 / 2}$ can be denoted as

$$
\mathbf{M}_{i}^{-\frac{1}{2}} \mathbf{Z}_{i} \mathbf{M}_{i}^{-\frac{1}{2}}=\tilde{\mathbf{U}}_{i} \tilde{\mathbf{\Sigma}}_{i} \tilde{\mathbf{U}}_{i}^{H}, \quad i=1,2
$$

where the eigenvalues in $\tilde{\boldsymbol{\Sigma}}_{i}$ are arranged in decreasing order.

Proof: See Appendix B.

Similar to [17], Lemma B.1 also implies that the optimal solution of (15) can be solved in an alternating manner, i.e., we can first solve problem (41) in Appendix B, and then update variables $\lambda_{i}$, for $i=1,2,3$, by using the subgradient-based method. The optimal solution of (15) can be obtained after convergence of the iteration. According to Lemma 2, solving the problem (41) can be simplified to solving the following power allocation problem:

$$
\begin{aligned}
\min _{\boldsymbol{\Sigma}_{Q 1}, \boldsymbol{\Sigma}_{Q 2}} & \left|\mathbf{I}_{N}+\boldsymbol{\Sigma}_{Q 1}\right|^{-\frac{1}{N}}+\left|\mathbf{I}_{N}+\boldsymbol{\Sigma}_{Q 2}\right|^{-\frac{1}{N}} \\
\text { s.t. } & \operatorname{Tr}\left\{\tilde{\boldsymbol{\Sigma}}_{1} \boldsymbol{\Sigma}_{Q 1}+\tilde{\boldsymbol{\Sigma}}_{2} \boldsymbol{\Sigma}_{Q 2}\right\} \leq P, \quad \boldsymbol{\Sigma}_{Q i} \succeq 0, \quad i=1,2
\end{aligned}
$$

where $P=\lambda_{1} P_{1}+\lambda_{2} P_{2}+\lambda_{3} P_{R}^{\prime}$. Let $\mathbf{X}_{Q 1}=\mathbf{I}_{N}+\boldsymbol{\Sigma}_{Q 1}$ and $\mathbf{X}_{Q 2}=\mathbf{I}_{N}+\Sigma_{Q 2}$; (17) can be reformed as

$$
\begin{aligned}
\min _{x_{Q 1}^{j}, x_{Q 2}^{j}} & \prod_{j=1}^{N} x_{Q 1}^{j}{ }^{-\frac{1}{N}}+\prod_{j=1}^{N} x_{Q 2}^{j}{ }^{-\frac{1}{N}} \\
\text { s.t. } & \sum_{j=1}^{N}\left(d_{1}^{j} x_{Q 1}^{j}+d_{2}^{j} x_{Q 1}^{j}\right) \leq \bar{P}, \quad x_{Q 1}^{j} \geq 1, x_{Q 2}^{j} \geq 1
\end{aligned}
$$

where $x_{Q i}^{j}$ is the $j$ th diagonal element of $\mathbf{X}_{Q i}, d_{i}^{j}$ is the $j$ th diagonal element of $\tilde{\boldsymbol{\Sigma}}_{i}$, and $\bar{P}=P+\operatorname{Tr}\left(\tilde{\boldsymbol{\Sigma}}_{2}+\tilde{\boldsymbol{\Sigma}}_{2}\right)$. Since (18) is convex, Karush-Kuhn-Tucker (KKT) conditions are sufficient and necessary for deriving the optimal solution. The Lagrange function of (18) can be written as

$$
\begin{aligned}
\mathcal{L}= & \prod_{j=1}^{N} x_{Q 1}^{j}-\frac{1}{N}+\prod_{j=1}^{N} x_{Q 2}^{j}{ }^{-\frac{1}{N}} \\
& +\lambda\left[\sum_{j=1}^{N}\left(d_{1}^{j} x_{Q 1}^{j}+d_{2}^{j} x_{Q 1}^{j}\right)-\bar{P}\right] \\
& +\sum_{j=1}^{N} \alpha_{j}\left(1-x_{Q 1}^{j}\right)+\sum_{j=1}^{N} \beta_{j}\left(1-x_{Q 2}^{j}\right)
\end{aligned}
$$


where $\lambda, \alpha_{j}$, and $\beta_{j}$ are nonnegative Lagrange variables related to the constraints in (18). By differentiating (19) with respect to $x_{Q i}^{j}$, the KKT conditions of (18) can be obtained as

$$
\begin{aligned}
\frac{\partial \mathcal{L}}{\partial x_{Q 1}^{j}}=- & \frac{1}{N} x_{Q 1}^{j}{ }^{-1} \prod_{l=1}^{N} x_{Q 1}^{l}{ }^{-\frac{1}{N}}+\lambda d_{1}^{j}-\alpha_{j}=0 \\
\frac{\partial \mathcal{L}}{\partial x_{Q 2}^{j}}=- & \frac{1}{N} x_{Q 2}^{j}{ }^{-1} \prod_{l=1}^{N} x_{Q 2}^{l}{ }^{\frac{1}{N}}+\lambda d_{2}^{j}-\beta_{j}=0 \\
\lambda\left(\sum_{j=1}^{N}\left(d_{1}^{j} x_{Q 1}^{j}+d_{2}^{j} x_{Q 1}^{j}\right)-\bar{P}\right) & =0 \\
\alpha_{j}\left(x_{Q 1}^{j}-1\right)=0, \beta_{j}\left(x_{Q 2}^{j}-1\right) & =0 .
\end{aligned}
$$

By multiplying (20) and (21) with $x_{Q 1}^{j}\left(x_{Q 2}^{j}\right)$ at both sides, we can obtain the following two equations:

$$
\begin{aligned}
& \frac{1}{N} \prod_{l=1}^{N} x_{Q 1}^{l}{ }^{-\frac{1}{N}}=\lambda d_{1}^{j} x_{Q 1}^{j}-\alpha_{j} x_{Q 1}^{j}=\lambda d_{1}^{j} x_{Q 1}^{j}-\alpha_{j} \\
& \frac{1}{N} \prod_{l=1}^{N} x_{Q 2}^{l-\frac{1}{N}}=\lambda d_{2}^{j} x_{Q 2}^{j}-\beta_{j} x_{Q 2}^{j}=\lambda d_{2}^{j} x_{Q 2}^{j}-\beta_{j}
\end{aligned}
$$

where the second equality of (24) and (25) is obtained by using (23). From (24) and (25), we find that $\lambda$ must be larger than zero. Since $\alpha_{j}$ and $\beta_{j}$ are two nonnegative Lagrange variables, to satisfy (23), if $\alpha_{j}$ or $\beta_{j}$ is larger than zero, we must have the following:

- If $\alpha_{j}>0$, then $x_{Q 1}^{j}=1$, and if $\beta_{j}>0$, then $x_{Q 2}^{j}=1$. On the other hand, if $\alpha_{j}$ or $\beta_{j}$ is equal to zero, we must obtain the following conclusion based on (24) and (25):

- If $\alpha_{j}=0$, then $d_{1}^{j} x_{Q 1}^{j}=a$ for $j=\left\{j \mid \alpha_{j}=0\right\}$, and if $\beta_{j}=0$, then $d_{2}^{j} x_{Q 2}^{j}=b$ for $j=\left\{j \mid \beta_{j}=0\right\}$.

Here, $a$ and $b$ are two positive scalars needed to be optimized. Since elements in the optimal solution $\mathbf{x}_{Q i}=$ $\left[x_{Q i}^{1}, x_{Q i}^{2}, \ldots, x_{Q i}^{N}\right]$ are arranged in increasing order as claimed in Lemma 2, if the optimal solution of (18) makes $x_{Q 1}^{j}=1, j=$ $1,2, \ldots, n$, and $x_{Q 2}^{j}=1, j=1,2, \ldots, m$, the optimization problem (18) can be simplified as

$$
\begin{aligned}
\min _{x_{Q 1}^{j}, x_{Q 2}^{j}} & \prod_{j=n+1}^{N} x_{Q 1}^{j}{ }^{-\frac{1}{N}}+\prod_{j=m+1}^{N} x_{Q 2}^{j}{ }^{-\frac{1}{N}} \\
\text { s.t. } & \sum^{N} d_{1}^{j} x_{Q 1}^{j}+\sum_{j=m+1}^{N} d_{2}^{j} x_{Q 1}^{j} \leq \tilde{P} \\
& x_{Q 1}^{j} \geq 1, \quad x_{Q 2}^{j} \geq 1
\end{aligned}
$$

where $\tilde{P}=\bar{P}-\sum_{j=1}^{n} d_{1}^{j}-\sum_{j=1}^{m} d_{2}^{j}$. Since $x_{Q i}^{j}$ in (26) should satisfy $d_{1}^{j} x_{Q 1}^{j}=a$ and $d_{2}^{j} x_{Q 2}^{j}=b$ as previously claimed, (26) can be further transformed into

$$
\begin{array}{ll}
\min _{a, b} & A \cdot a^{-\frac{N-n}{N}}+B \cdot b^{-\frac{N-m}{N}} \\
\text { s.t. } & (N-n) a+(N-m) b \leq \tilde{P} \\
& a \geq d_{1}^{n+1}, \quad b \geq d_{2}^{m+1}
\end{array}
$$

where $A=\prod_{j=n+1}^{N} d_{1}^{j^{1 / N}}$, and $B=\prod_{j=m+1}^{N} d_{2}^{j^{1 / N}}$. Again, by using the Lagrange function of (27) given by

$$
\begin{aligned}
\mathcal{L}=J_{1}+J_{2}+\gamma((N-n) a+(N-m) b-\tilde{P}) & \\
& +\mu\left(d_{1}^{n+1}-a\right)+\nu\left(d_{2}^{m+1}-a\right)
\end{aligned}
$$

where $J_{1}=A \cdot a^{-N-n / N}$ and $J_{2}=B \cdot b^{-N-m / N}$, the KKT conditions are obtained as

$$
\begin{aligned}
\frac{\partial J_{1}}{\partial a}+\gamma(N-n)-\mu & =0 \\
\frac{\partial J_{2}}{\partial b}+\gamma(N-m)-\nu & =0 \\
\gamma((N-n) a+(N-m) b-\tilde{P}) & =0 \\
\mu\left(a-d_{1}^{n+1}\right)=0, \quad \nu\left(b-d_{2}^{m+1}\right) & =0 .
\end{aligned}
$$

Combining (29) with the observation that the optimal solution in (27) must make the power constraint active; we can derive the following three points, which include the optimal solution:

Case I: If $\mu \neq 0$, then

$$
a=d_{1}^{n+1}, \quad b=\frac{\tilde{P}-(N-n) a}{N-m} .
$$

Case II: If $\nu \neq 0$, then

$$
b=d_{2}^{m+1}, \quad a=\frac{\tilde{P}-(N-m) b}{N-n} .
$$

Case III: If $\mu=0, \nu=0$, then $(a, b)$ satisfies

$$
\begin{aligned}
& (N-m) \frac{\partial J_{1}}{\partial a}=(N-n) \frac{\partial J_{2}}{\partial b} \\
& (N-n) a+(N-m) b=\tilde{P} .
\end{aligned}
$$

By comparing these points, we can obtain the optimal solution of (27). Then, using $a=d_{1}^{j} x_{Q 1}^{j}$ and $b=d_{2}^{j} x_{Q 2}^{j}$ in (26), we finally obtain the optimal solution of (18) under the assumption that $x_{Q 1}^{j}=1$ for $j=1,2, \ldots, n$ and $x_{Q 2}^{j}=1$ for $j=1,2, \ldots, m$. By trying all possible cases of $(n, m)$, we can find the optimal solution of (17) and then using Lemma 2 to obtain the optimal solution of (41). Note that, in (41), there exists variable $\lambda_{i}$, which needs to be updated by making use of the subgradient-based method.

In summary, the overall algorithm to optimally solve (15) is outlined as follows:

- Repeat

- Solve the problem (17) for fixed $\lambda_{i}(n), i=1,2,3$

— For $n, m=0,1,2, \ldots, N$, do

- Solve (26) under the assumption $x_{Q 1}^{j}=1, j=1, \ldots, n$, $x_{Q 2}^{j}=1, j=1, \ldots, m$ by using Cases I, II, and III in (30)-(32).

- End

- Choose an $(n, m)$, which gets the minimal value of the objective function of (18), and obtain the optimal 
solution of (17). Then, use Lemma 2 to get the optimal $\mathbf{Q}_{1}\left(\lambda_{i}(n)\right)$ and $\mathbf{Q}_{2}\left(\lambda_{i}(n)\right)$.

- Update variables $\lambda_{1}(n), \lambda_{2}(n)$, and $\lambda_{3}(n)$ using the subgradient-based method ${ }^{3}$

$$
\begin{aligned}
\lambda_{1}(n+1)= & \lambda_{1}(n)-\Delta_{n}\left(P_{1}-\operatorname{Tr}\left(\mathbf{Q}_{1}\left(\lambda_{i}(n)\right)\right)\right) \\
\lambda_{2}(n+1)= & \lambda_{2}(n)-\Delta_{n}\left(P_{2}-\operatorname{Tr}\left(\mathbf{Q}_{2}\left(\lambda_{i}(n)\right)\right)\right) \\
\lambda_{3}(n+1)= & \lambda_{3}(n)-\Delta_{n}\left(P_{R}-\operatorname{Tr}\left(\mathbf{N}_{1} \mathbf{Q}_{1}\left(\lambda_{i}(n)\right)\right)\right. \\
& \left.\left.\left.+\mathbf{N}_{2} \mathbf{Q}_{2}\left(\lambda_{i}(n)\right)\right)\right)\right) .
\end{aligned}
$$

- Until the termination criterion is satisfied.

By decomposing $\mathbf{Q}_{i}$, we can get the optimal $\mathbf{F}_{i}^{\mathrm{lb}}$. Note that, until now, the optimal $\mathbf{F}_{i}^{\mathrm{lb}}, i=1,2$ are obtained based on the lower bound of (10). Next, we show that suitably chosen $\mathbf{U}_{i}$ can achieve this lower bound, which makes the preceding design indeed optimal. Before that, we need to be reminded that $E_{i}$ in (10) achieving the lower bound $E_{i}^{\mathrm{lb}}$ in (13) only occurs when [13]

$$
\mathbf{U}_{i}\left(\mathbf{I}_{K}+\mathbf{F}_{\bar{i}}^{H} \mathbf{H}_{\bar{i}}^{H} \mathbf{P}^{H} \mathbf{G}_{i}^{H} \mathbf{R}_{i}^{-1} \mathbf{G}_{i} \mathbf{P} \mathbf{H}_{\bar{i}} \mathbf{F}_{\bar{i}}\right)^{-1} \mathbf{U}_{i}^{H}=q \mathbf{I}_{N}
$$

where $q=\left|\mathbf{I}_{K}+\mathbf{D}_{i}^{H} \mathbf{R}_{i}^{-1} \mathbf{D}_{i}\right|^{-1 / N}$. Note that multiplying any unitary matrix at the right side of $\mathbf{F}_{i}^{\mathrm{lb}}$ does not change the value of $E^{\mathrm{lb}}$ in (13). Letting $\mathbf{S}_{i}$ be a unitary matrix, we have

$$
\begin{aligned}
E_{\bar{i}}= & \operatorname{Tr}\left\{\mathbf { U } _ { i } \left(\mathbf{I}_{K}+\mathbf{S}_{i}^{H} \mathbf{F}_{\bar{i}}^{\mathrm{lb}}{ }^{H} \mathbf{H}_{\bar{i}}^{H} \mathbf{P}^{H}\right.\right. \\
& \left.\left.\times \mathbf{G}_{i}^{H} \mathbf{R}_{i}^{-1} \mathbf{G}_{i} \mathbf{P} \mathbf{H}_{i} \mathbf{F}_{\bar{i}}^{\mathrm{lb}} \mathbf{S}_{i}\right)^{-1} \mathbf{U}_{i}^{H}\right\} \\
= & \operatorname{Tr}\left\{\mathbf{U}_{i} \mathbf{S}_{i}^{H} \mathbf{A}_{i} \mathbf{S}_{i} \mathbf{U}_{i}^{H}\right\}
\end{aligned}
$$

where $\mathbf{A}_{i}=\left(\mathbf{I}_{K}+\mathbf{F}_{\bar{i}}^{\mathrm{lb}}{ }^{H} \mathbf{H}_{\bar{i}}^{H} \mathbf{P}^{H} \mathbf{G}_{i}^{H} \mathbf{R}_{i}^{-1} \mathbf{G}_{i} \mathbf{P} \mathbf{H}_{\bar{i}} \mathbf{F}_{\bar{i}}^{\mathrm{lb}}\right)^{-1}$. To make (33) succeed, we have

$$
\mathbf{U}_{i} \mathbf{S}_{i}^{H} \mathbf{A}_{i}^{1 / 2}=\sqrt{q} \mathbf{V}_{i}
$$

where $\mathbf{V}_{i}$ can be any unitary matrix. Equation (35) further leads to

$$
\mathbf{V}_{i}^{H} \mathbf{U}_{i}=\sqrt{q} \mathbf{A}_{i}^{-1 / 2} \mathbf{S}_{i} .
$$

By using the equal diagonal QR decomposition method proposed in [19], we can always find a unitary matrix $\mathbf{S}_{i}$ that makes the QR decomposition of $\sqrt{q} \mathbf{A}_{i}^{-1 / 2} \mathbf{S}_{i}$ have equal diagonal upper triangle matrix $\mathbf{U}_{i}$. The optimal $\mathbf{F}_{i}$ in (12) can be derived as $\mathbf{F}_{i}=\mathbf{F}_{i}^{\mathrm{lb}} \mathbf{S}_{i}$. Then, we substitute the derived $\mathbf{U}_{i}$ and $\mathbf{F}_{i}$ into (9) to get the optimal feedforward matrix $\mathbf{W}_{i}$.

Remark: Since we have proven the convexity of (15), the existing advanced software package CVX [20] can also be applied to obtain the optimal solution. The advantage of the proposed algorithm over CVX is that it can obtain more accurate solution in the low signal-to-noise ratio (SNR) regime since,

\footnotetext{
${ }^{3}$ The subgradient can be found as in [17], and the step size can be chosen as in [18].
}

by using Lemma 2, the not-yet-determined elements in (15) have been reduced from $2 N^{2}$ to $2 N+3$. This is also verified in the simulation results. In addition, the proposed algorithm is necessary in certain scenarios where the advanced software package is not available.

\section{B. Joint Nonlinear Precoding Design}

In this section, we consider the joint nonlinear precoding design to further incorporate the relay precoding. Although the algorithm proposed in Section III-A enables the optimal design of the nonlinear source precoding for a fixed-relay precoder, it is not easy to design all the optimal precoding and decoding matrices jointly due to the nonconvexity of (8), where there are seven matrices to be optimized. To find an efficient way to approach the optimal solution, we next propose two iterative algorithms to decompose the primal problem into two subproblems and solve each of them in an alternating manner.

1) Algorithm I: In the first subproblem, we aim to solve the transmit matrices $\mathbf{F}_{i}$, for $i=1,2$, and four receive matrices $\mathbf{W}_{i}$ and $\mathbf{B}_{i}$ (i.e., $\mathbf{U}_{i}$ ), for $i=1,2$, for a given relay precoder $\mathbf{P}$. Note that this subproblem can be transformed into (12) and then optimally solved by using the algorithm provided in Section III-A. Thus, the optimal $\mathbf{F}_{i}, \mathbf{W}_{i}, \mathbf{B}_{i}$, for $i=1,2$, can be obtained in this subproblem.

In the second subproblem, we shall update the relay precoder $\mathbf{P}$ in (8) for fixed $\mathbf{F}_{i}, \mathbf{W}_{i}, \mathbf{B}_{i}$, for $i=1,2$. After applying the circular property of trace operator $\operatorname{Tr}\{\mathbf{A B}\}=\operatorname{Tr}\{\mathbf{B A}\}$ on (7), this subproblem can be rewritten as

$$
\begin{gathered}
\min _{\mathbf{P}} \sum_{i=1}^{2} \operatorname{Tr}\left\{\mathbf{G}_{i}^{H} \mathbf{W}_{i}^{H} \mathbf{W}_{i} \mathbf{G}_{i}^{H} \mathbf{P} \mathbf{R}_{i} \mathbf{P}-\mathbf{W}_{i} \mathbf{G}_{i} \mathbf{P} \mathbf{H}_{i} \mathbf{F}_{\bar{i}} \mathbf{U}_{i}^{H}\right. \\
-\mathbf{U}_{i} \mathbf{F}_{\bar{i}}^{H} \mathbf{H}_{\bar{i}}^{H} \mathbf{P}^{H} \mathbf{G}_{i}^{H} \mathbf{W}_{i}^{H} \\
\left.+\sigma_{i}^{2} \mathbf{W}_{i} \mathbf{W}_{i}^{H}+\mathbf{U}_{i} \mathbf{U}_{i}^{H}\right\}
\end{gathered}
$$

where $\mathbf{R}_{i}=\mathbf{H}_{\bar{i}} \mathbf{F}_{\bar{i}} \mathbf{F}_{\bar{i}}^{H} \mathbf{H}_{\bar{i}}^{H}+\sigma_{R}^{2} \mathbf{I}_{M}$. In [7] and [8], we have proven that (37) is convex with respect to $\mathbf{P}$, and the optimal $\mathbf{P}$ can be obtained by using the following lemma:

Lemma 3: The optimal closed-form $\mathbf{P}$ of (37) can be derived as

$$
\begin{aligned}
\mathbf{P}=\operatorname{mat}\{[ & \left(\mathbf{H}_{1} \mathbf{F}_{1} \mathbf{F}_{1}^{H} \mathbf{H}_{1}^{H}+\sigma_{2}^{2} \mathbf{I}_{N}\right)^{T} \otimes\left(\mathbf{G}_{2}^{H} \mathbf{W}_{2}^{H} \mathbf{W}_{2} \mathbf{G}_{2}\right) \\
& +\left(\mathbf{H}_{2} \mathbf{F}_{2} \mathbf{F}_{2}^{H} \mathbf{H}_{2}^{H}+\sigma_{1}^{2} \mathbf{I}_{N}\right)^{T} \otimes\left(\mathbf{G}_{1}^{H} \mathbf{W}_{1}^{H} \mathbf{W}_{1} \mathbf{G}_{1}\right) \\
& \left.\left.+\eta \mathbf{O}_{1}^{T} \otimes \mathbf{I}_{M}\right]^{-1} \operatorname{vec}\left(\mathbf{O}_{2}\right)\right\}
\end{aligned}
$$

where $\mathbf{O}_{1}=\mathbf{H}_{1} \mathbf{F}_{1} \mathbf{F}_{1}^{H} \mathbf{H}_{1}^{H}+\mathbf{H}_{2} \mathbf{F}_{2} \mathbf{F}_{2}^{H} \mathbf{H}_{2}^{H}+\sigma_{R}^{2} \mathbf{I}_{N}$, and $\mathbf{O}_{2}=$ $\mathbf{G}_{1}^{H} \mathbf{W}_{1}^{H} \mathbf{U}_{1} \mathbf{F}_{2}^{H} \mathbf{H}_{2}^{H}+\mathbf{G}_{2}^{H} \mathbf{W}_{2}^{H} \mathbf{U}_{2} \mathbf{F}_{1}^{H} \mathbf{H}_{1}^{H}$. The optimal $\eta$ is bounded within $\left[0, \sqrt{\mathbf{O}_{2} \mathbf{O}_{1}^{-1} \mathbf{O}_{2}^{H} / P_{R}}\right]$ and can be found by using bisection search to satisfy the KKT conditions of (37).

Since the proof of Lemma 3 is similar to the proof of Lemma 2 in [7] and [8], thus we omit it for brevity. 
Overall, Algorithm I is summarized as follows:

\section{- Repeat}

- Solve (12) with $\mathbf{P}$ fixed to get the optimal $\mathbf{F}_{i}$ and $\mathbf{U}_{i}$, for $i=1,2$, and then, get $\mathbf{W}_{i}$ by using (9).

- Solve the relay precoder $\mathbf{P}$ in (37) for fixed $\mathbf{F}_{i}, \mathbf{W}_{i}, \mathbf{B}_{i}$, for $i=1,2$, by using (38).

- Until the termination criterion is satisfied.

Lemma 4: The proposed iterative precoding design algorithm, i.e., Algorithm I, is convergent, and the limit point of the iteration is a stationary point of (8).

Proof: Since, in the proposed algorithm, the solution for each subproblem is optimal, the Total-MSE is decreased after each iteration. Meanwhile, the Total-MSE is lower bounded (at least by zero). Hence, the proposed algorithm is convergent. It further means that there must exist a limit point denoted as $\left\{\overline{\mathbf{F}}_{i}, \overline{\mathbf{P}}, \overline{\mathbf{W}}_{i}, \overline{\mathbf{U}}_{i}, i=1,2\right\}$ after the convergence. At the limit point, the solution will not change if we continue the iteration. Otherwise, the Total-MSE can be further decreased, and it contradicts the assumption of convergence. Since $\left\{\overline{\mathbf{F}}_{i}, \overline{\mathbf{W}}_{i}, \overline{\mathbf{U}}_{i}, i=\right.$ $1,2\}$ and $\overline{\mathbf{P}}$ are local minimizers for each subproblem, we have

$$
\begin{array}{r}
\operatorname{Tr}\left\{\nabla_{\mathbf{X}} J(\overline{\mathbf{X}} ; \overline{\mathbf{P}}, i=1,2)^{T}(\mathbf{X}-\overline{\mathbf{X}})\right\} \\
\geq 0 \\
\operatorname{Tr}\left\{\nabla_{\mathbf{P}} J\left(\overline{\mathbf{P}} ; \overline{\mathbf{F}}_{i}, \overline{\mathbf{W}}_{i}, \overline{\mathbf{U}}_{i}, i=1,2\right)^{T}(\mathbf{P}-\overline{\mathbf{P}})\right\} \geq 0
\end{array}
$$

where $\mathbf{X}=\left[\mathbf{F}_{1}, \mathbf{F}_{2}, \mathbf{W}_{1}, \mathbf{W}_{2}, \mathbf{U}_{1}, \mathbf{U}_{2}\right]$, and $J=E_{1}+E_{2}$. Summing up the two preceding equations, we get

$$
\operatorname{Tr}\left\{\nabla_{\mathbf{Y}} J(\overline{\mathbf{Y}})^{T}(\mathbf{Y}-\overline{\mathbf{Y}})\right\} \geq 0
$$

where $\mathbf{Y}=\left[\mathbf{F}_{1}, \mathbf{F}_{2}, \mathbf{P}, \mathbf{U}_{1}, \mathbf{U}_{2}, \mathbf{W}_{1}, \mathbf{W}_{2}\right]$. Result (39) implies the stationarity of $\overline{\mathbf{Y}}$ of (8) by definition.

We now provide some discussions on the design complexity of Algorithm I. In general, it consists of two parts, i.e., solving the source precoders and solving the relay precoder. For solving the source precoders, almost $n_{\text {ite }}^{s}(N+1)^{2}$ steps are needed to find the final solution, where $n_{\text {ite }}^{s}$ denotes the required iterations. While for solving the relay precoder, since bisection search is used to find the optimal $\eta$ in (38), $\left\lceil\log _{2}(\bar{N} / e)\right\rceil$ iterations are required to obtain the final relay precoder by using Lemma 3, where $\bar{N}=\sqrt{\mathbf{O}_{2} \mathbf{O}_{1}^{-1} \mathbf{O}_{2}^{H} / P_{R}}$ is a preset search upper bound of $\eta$, and $e$ is the error precision for the convergence of bisection search. Thus, the overall design complexity of Algorithm I can be approximated as

$$
L=O\left(n_{\text {ite }}\left(n_{\text {ite }}^{s}(N+1)^{2}+\left\lceil\log _{2}(\bar{N} / e)\right\rceil\right)\right)
$$

where $n_{\text {ite }}$ denotes the required iterations in Algorithm I.

2) Algorithm II: It is easy to find that the high design complexity of Algorithm I is due to the fact that no closedform solution is available when solving the first subproblem of Algorithm I. By taking a closer look at the optimization problem (14), we find that the difficulty lies at existing relay power constraint such that solving (14) becomes quite different from solving the nonlinear precoding design problem in traditional point-to-point systems. Therefore, to reduce the design complexity, we next propose a simplified iterative algorithm by
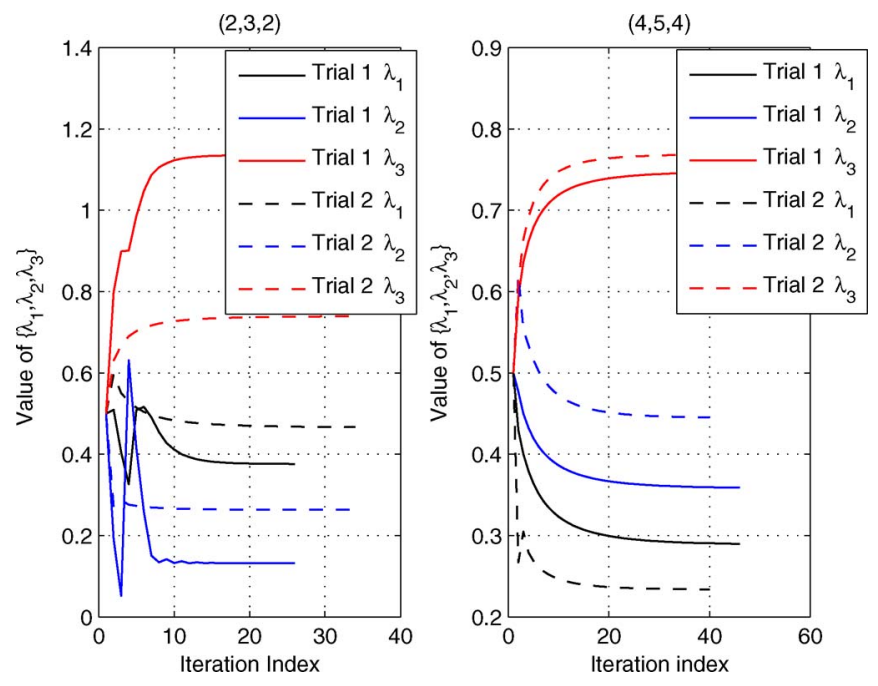

Fig. 2. Iterations required to update $\left\{\lambda_{1}, \lambda_{2}, \lambda_{3}\right\}$ for $(2,3,2)$ and $(4,5,4)$ MIMO two-way relay systems at $\rho_{1}=\rho_{2}=\rho_{R}=10 \mathrm{~dB}$.

discarding the relay power constraint when solving (14). Then, solving (14) is simplified to solve the following problem:

$$
\begin{array}{cl}
\max _{\mathbf{F}_{i}} & \left|\mathbf{I}_{N}+\mathbf{F}_{i}^{\mathrm{lb} H} \mathbf{M}_{i} \mathbf{F}_{i}^{\mathrm{lb}}\right|^{1 / N} \\
\text { s.t. } & \operatorname{Tr}\left(\mathbf{F}_{i}^{\mathrm{lb}} \mathbf{F}_{i}^{\mathrm{lb} H}\right) \leq P_{i} .
\end{array}
$$

The closed-form solution derived in [13] can be used to independently obtain $\mathbf{F}_{1}^{\mathrm{lb}}$ and $\mathbf{F}_{2}^{\mathrm{lb}}$, which can significantly reduce the computational complexity. Therefore, different from Algorithm I, in the first subproblem of Algorithm II, we directly solve (40) instead of solving (14). Note that, since Algorithm II is heuristic, the convergence of iteration cannot be strictly proven, whereas, as verified by our simulation, we find that the algorithm is convergent in most cases. In some special scenarios, we terminate the iteration when the iteration number exceeds a preset threshold.

\section{Simulation Results and Discussions}

In this section, some simulation results are presented to evaluate the proposed precoding designs. The channels are modeled as Rayleigh fading, i.e., the elements of each channel matrix are complex Gaussian random variables with zero mean and unit variance. The noise powers at all destinations are set to be the same, i.e., $\sigma_{1}^{2}=\sigma_{2}^{2}=\sigma_{R}^{2}=\sigma^{2}$. The average SNRs for the MAC phase and BC phase are defined as $\rho_{1}=P_{1} / \sigma^{2}, \rho_{2}=$ $P_{2} / \sigma^{2}$, and $\rho_{R}=P_{R} / \sigma^{2}$, respectively. The average bit error rate (BER) using quadrature phase-shift keying modulation is simulated. If not specified otherwise, the previous decoded symbols are used for the subsequent interference subtraction at the MMSE-DFE receiver.

In Fig. 2, we show the convergence behavior of updating $\left\{\lambda_{1}, \lambda_{2}, \lambda_{3}\right\}$ for two random channel realizations. We find that, in general, the update of $\left\{\lambda_{1}, \lambda_{2}, \lambda_{3}\right\}$ converges fast. To explicitly show how many iterations are needed on average, we plot the average iteration number in Fig. 3 as a function of SNR. It is observed that almost 150 iterations are enough for all the SNR scenarios. 


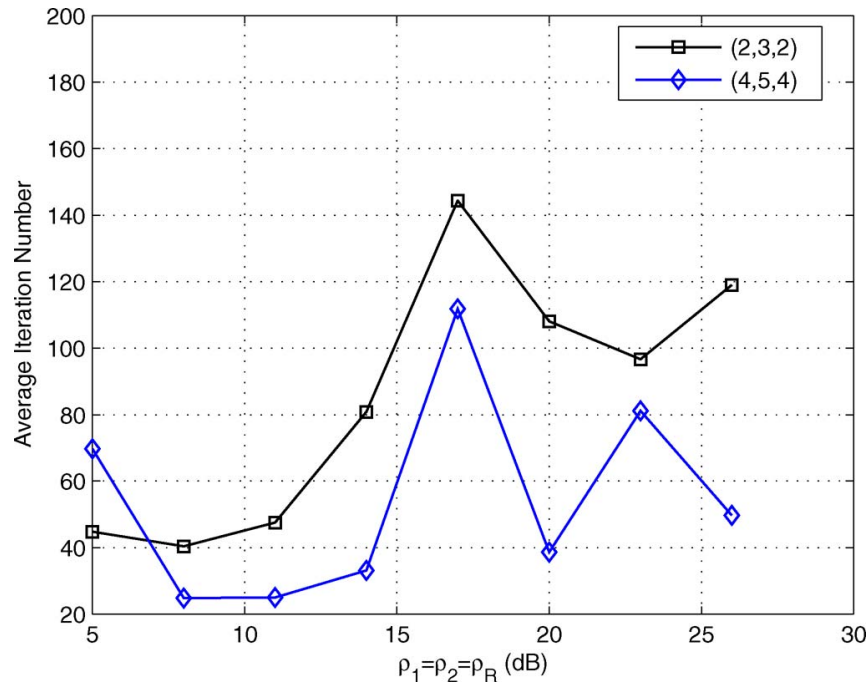

Fig. 3. Average iterations required to update $\left\{\lambda_{1}, \lambda_{2}, \lambda_{3}\right\}$.

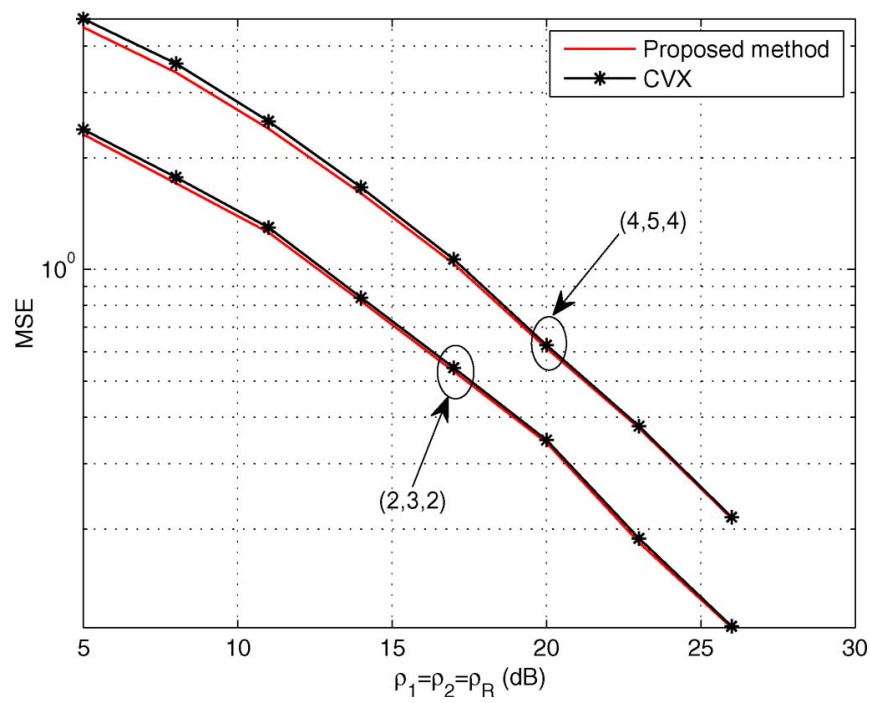

Fig. 4. Performance comparison of the nonlinear source precoding design by using the proposed method and CVX.

Fig. 4 shows the MSE comparison of the nonlinear source precoding design by using the proposed method and CVX [20]. It is observed that, although they obtain the same performance in the high-SNR regime, the proposed method performs slightly better than CVX in the low-SNR regime. The reason is that, in our proposed method, we have used the optimal precoding structure derived in Lemma 2, which reduces the not-yetdetermined elements in (15) from $2 N^{2}$ to $2 N+3$. Thereby, the proposed method can obtain more accurate solution than CVX.

In Fig. 5, we show the MSE and BER comparisons among three proposed designs for $(2,3,2)$ two-way relay system. The curves first show that the comparison results for MSE and BER are the same. It is also found that the joint design can significantly increase the system performance compared with the source precoding design, and two joint precoding designs almost obtain similar performance. This observation can also be found in Fig. 6, where the performance of Algorithms I and II is compared under several antenna configurations. The intuitive explanation for this observation is that, although the solution
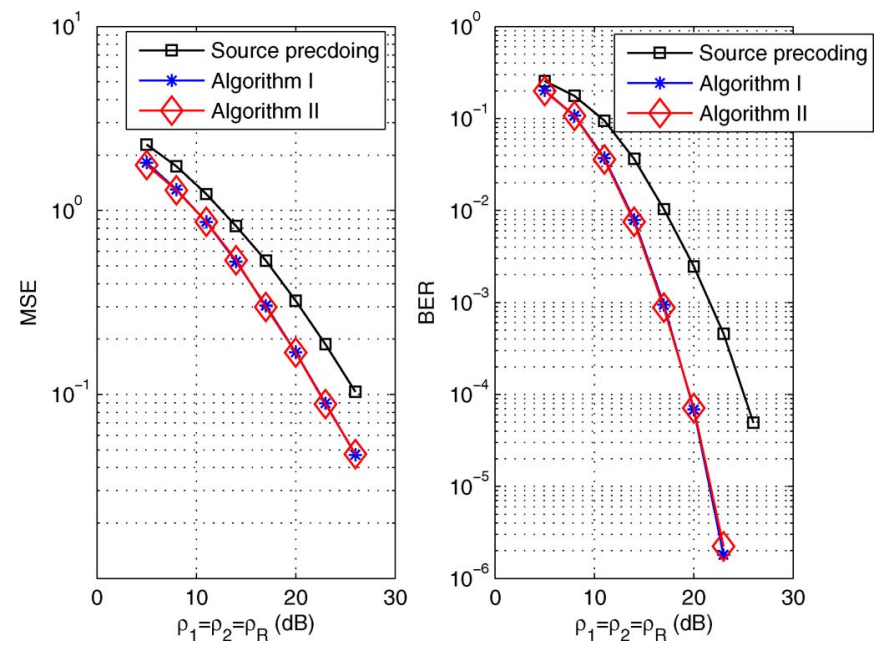

Fig. 5. MSE and BER comparison of three proposed designs for $(2,3,2)$ twoway relay system.

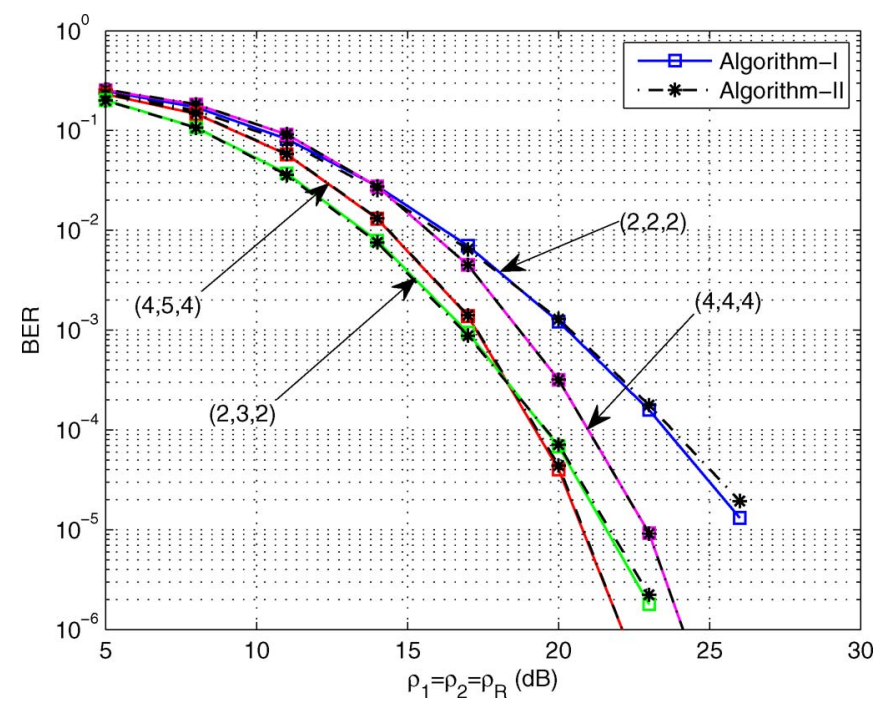

Fig. 6. Performance comparison between Algorithm I and Algorithm II.

of precoding matrix $\mathbf{F}_{i}$ during the first step in Algorithm II is suboptimal by ignoring the relay power constraint, the relay power violation can be compensated in the second step by optimizing the relay precoder. This way, the relay power violation may only occur at the beginning of the iteration. As the iterative procedure converges, the solved source precoders begin satisfying the relay power constraint. Therefore, the obtained final solution from Algorithm II may be close to that from Algorithm I. Thus, Algorithm I and Algorithm II can obtain similar performance. The comparison result also implies that, in practical implementation, we can discard the relay power constraint when solving the source precoder in the first subproblem to highly reduce the design complexity. In the following, if not specified otherwise, the nonlinear joint precoding means using Algorithm II to perform the joint precoding design.

Fig. 7 shows performance comparison for the perfect previous symbol subtraction and the decoded previous symbol subtraction at the MMSE-DFE receiver. It is found that the propagation of the detection error only slightly increases the system BER. The performance of the proposed precoding 


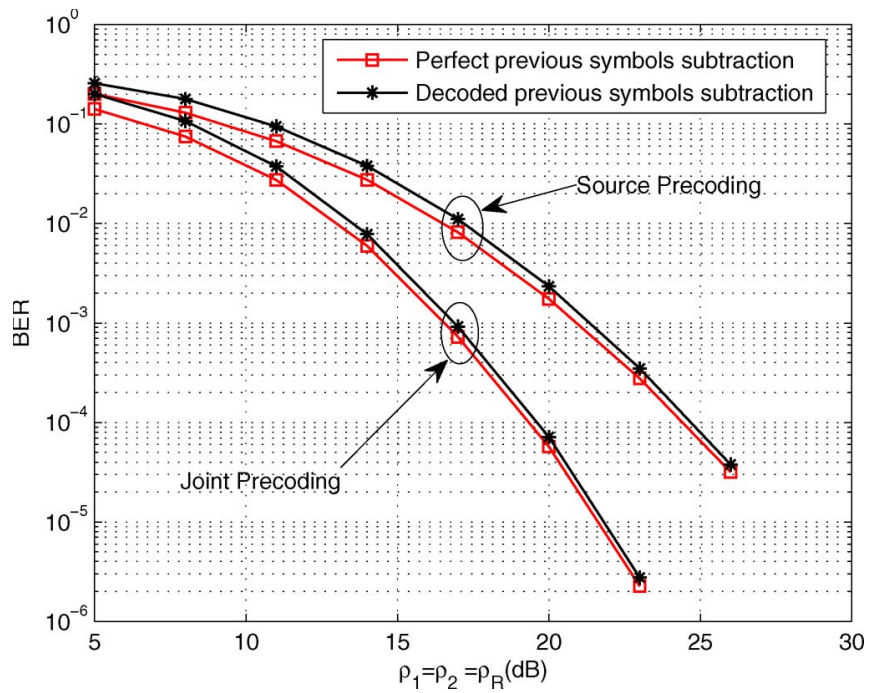

Fig. 7. Performance comparison of perfect previous symbols subtraction and decoded previous symbol subtraction for $(2,3,2)$ two-way relay system.
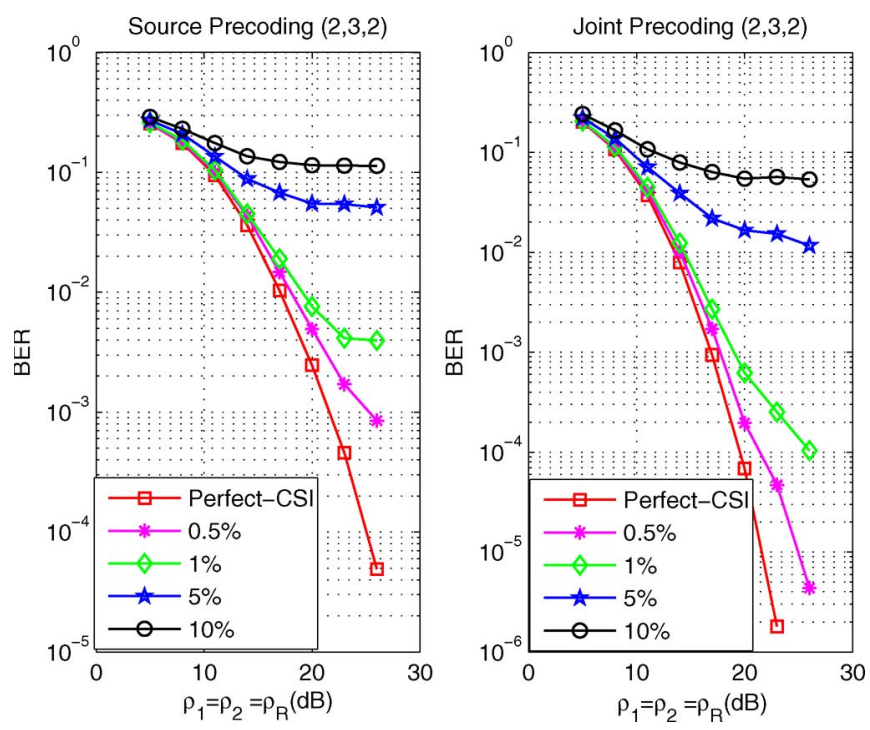

Fig. 8. Performance of the proposed precoding designs over imperfect CSI.

designs over imperfect CSI is also shown in Fig. 8, where $N \%$ means the elements of each channel error following the distribution of $\mathcal{C} \mathcal{N}(0,1 \cdot N \%)$. The plots in Fig. 8 show that the nonlinear precoding is sensitive to the CSI error, and system performance can be greatly degraded if the CSI error is large.

Next, we aim to compare the performance of proposed nonlinear precoding designs with the linear precoding designs proposed in [7] and [8]. Overall, five schemes are simulated.

1) Scheme I: nonlinear source precoding for a fixed-relay precoder, which is simply set as $\mathbf{P}=\sqrt{P_{R} / \operatorname{Tr}\left(P_{1} \mathbf{H}_{1} \mathbf{H}_{1}^{H}+P_{2} \mathbf{H}_{2} \mathbf{H}_{2}^{H}+\sigma_{R}^{2} \mathbf{I}_{M}\right)} \mathbf{I}_{M} ; 4$

2) Scheme II: nonlinear joint precoding;

${ }^{4}$ Using this relay precoder implies that the relay node does not need to know which precoders have been used at two source nodes. Moreover, since the designed source precoders in Scheme I satisfy the relay power constraint, the relay does not need to scale the received signal in each data transmission, which can reduce the relay processing complexity.

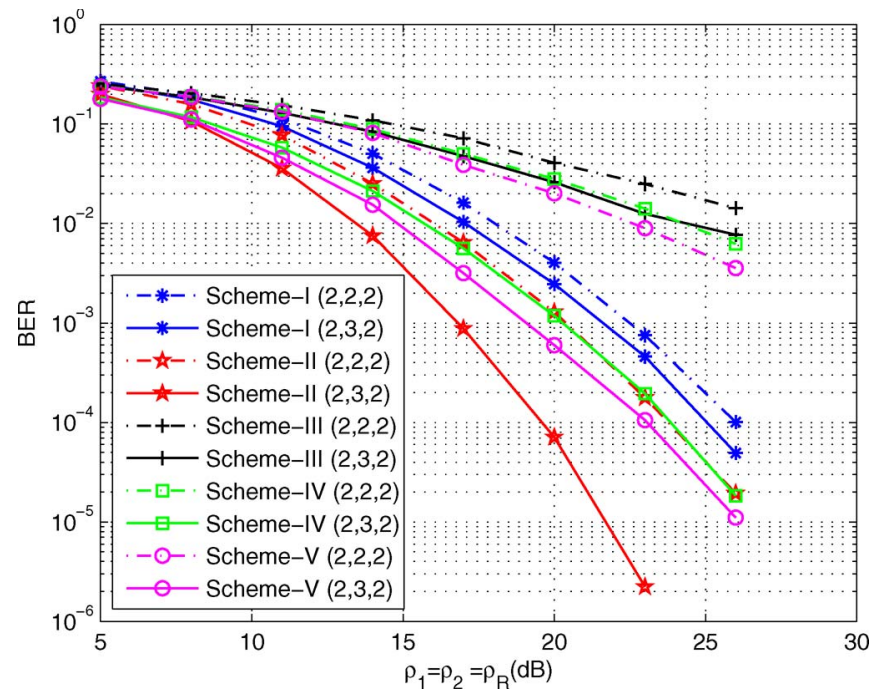

Fig. 9. Performance comparison for different precoding schemes at $N=2$.

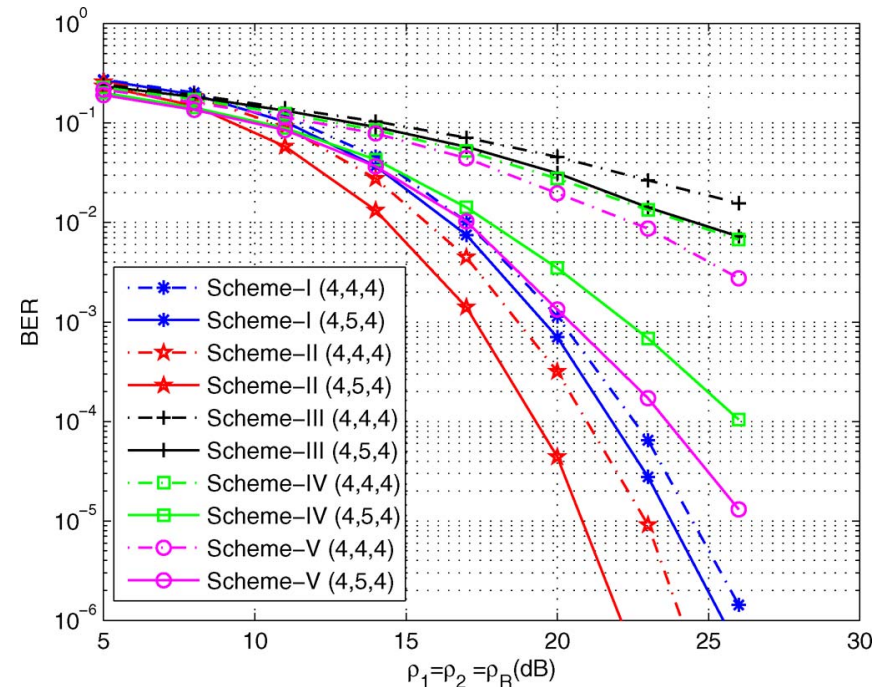

Fig. 10. Performance comparison for different precoding schemes at $N=4$.

3) Scheme III: linear source precoding for a fixed-relay precoder $\mathbf{P}$ as in Scheme I;

4) Scheme IV: linear relay precoding for fixed-source precoders $\mathbf{F}_{1}=\sqrt{P_{1} / N} \mathbf{I}_{N}$ and $\mathbf{F}_{2}=\sqrt{P_{2} / N} \mathbf{I}_{N}$;

5) Scheme $\mathrm{V}$ : linear joint precoding.

For the linear precoding Schemes III, IV, and V, we apply the iterative precoding designs proposed in [7] and [8]. Note that we do not consider the nonlinear relay precoding since the nonlinear precoding design needs to jointly design the precoding matrix at the source node and the decoding matrices at the destination. It is not feasible to only design the relay precoding matrix and destination decoding matrices in the considered twoway relay system.

In Figs. 9 and 10, we consider two cases with $N=2$ and $N=4$, respectively. From the plots, we find that, for the nonlinear precoding, Scheme II significantly outperforms Scheme I, as expected, since the relay precoding is incorporated in Scheme II. Furthermore, when increasing the relay 
antenna number, the improved performance gain of Scheme I is obviously less than Scheme II. On the other hand, for the linear precoding schemes, we find that source-only precoding (Scheme III) performs much worse than relay-only precoding (Scheme IV), which can get most of the performance gain of the joint precoding scheme. This observation means that, in the two-way relay system, precoding at the relay node is very effective. For the comparison between nonlinear precoding and linear precoding, we observe that the joint nonlinear precoding scheme (Scheme II) significantly outperforms the joint linear precoding scheme (Scheme V). This observation is expected due to applying more advanced successive interference cancelation receivers for the nonlinear precoding. The more interesting comparison is between the nonlinear source precoding and the linear relay precoding. We find that, when $N=2$, although Scheme I $(2,2,2)$ outperforms Scheme IV $(2,2,2)$, when equipping one more antenna at the relay node, Scheme IV $(2,3,2)$ begins to outperform Scheme I $(2,3,2)$, which means that, in this case, applying linear precoding at the relay node is more efficient than applying complex nonlinear source precoding. However, this observation does not occur if we equip one more relay antenna for the case $N=4$ in Fig. 10. The main reason is that, when more data streams are transmitted from two sources, Scheme I can obtain more performance gain by using the nonlinear MMSE-DFE receiver.

\section{CONCLUSION}

In this paper, we have considered the nonlinear precoding design for MIMO AF two-way relay systems. We have first studied the nonlinear source precoding design for a fixed-relay precoder. This problem has been proven to be convex, and an efficient algorithm has been proposed to get the optimal solution. We have then studied the more complex nonlinear joint precoding design. Due to the nonconvexity of this problem, we have proposed two iterative algorithms to find the final solution. Simulation results have verified the efficiency of the proposed nonlinear joint precoding design over the existing linear joint precoding design.

\section{APPENDIX A \\ PROOF OF LEMMA 1}

Since the feasible set of (15) is convex, to prove the convexity of (15), we only need to verify that the objective function is convex. To achieve this goal, we first show that $f_{i}\left(\mathbf{Q}_{i}\right)=\mid \mathbf{I}_{N}+$ $\left.\mathbf{M}_{i} \mathbf{Q}_{i}\right|^{1 / N}$, for $i=1,2$, is concave. For brevity, we next take $f_{1}\left(\mathbf{Q}_{1}\right)$ as an example. According to [21], we can prove the concavity of $f_{1}\left(\mathbf{Q}_{1}\right)$ by considering an arbitrary linear, given by $\mathbf{Q}_{1}=\mathbf{Z}+t \mathbf{V}$, where $\mathbf{Z} \in \mathbb{S}_{+}^{N}, \mathbf{V} \in \mathbb{S}^{N}$ and $\mathbf{Z}+t \mathbf{V} \in \mathbb{S}_{+}^{N}$. By defining $g(t)=f_{1}(\mathbf{Z}+t \mathbf{V})$, we have

$$
\begin{aligned}
g(t) & =\left|\mathbf{I}_{N}+\mathbf{M}_{1}(\mathbf{Z}+t \mathbf{V})\right|^{\frac{1}{N}} \\
& =\left|\mathbf{I}_{N}+\mathbf{M}_{1}^{\frac{1}{2}} \mathbf{Z} \mathbf{M}_{1}^{\frac{1}{2}}+t \mathbf{M}_{1}^{\frac{1}{2}} \mathbf{V} \mathbf{M}_{1}^{\frac{1}{2}}\right|^{\frac{1}{N}} \\
& =|\mathbf{A}+t \mathbf{B}|^{\frac{1}{N}}
\end{aligned}
$$

where $\mathbf{A}=\mathbf{I}_{N}+\mathbf{M}_{1}^{1 / 2} \mathbf{Z} \mathbf{M}_{1}^{1 / 2}$, and $\mathbf{B}=\mathbf{M}_{1}^{1 / 2} \mathbf{V M}_{1}^{1 / 2}$. Then, we obtain

$$
\begin{aligned}
\frac{d^{2} g(t)}{d t^{2}}=\frac{1}{N^{2}}|\mathbf{A}+t \mathbf{B}|^{\frac{1}{N}}\left[\operatorname{Tr}^{2}\left\{(\mathbf{A}+t \mathbf{B})^{-1} \mathbf{B}\right\}\right. \\
\left.-N \operatorname{Tr}\left\{(\mathbf{A}+t \mathbf{B})^{-1} \mathbf{B}(\mathbf{A}+t \mathbf{B})^{-1} \mathbf{B}\right\}\right]
\end{aligned}
$$

We next show that $\operatorname{Tr}^{2}\left\{(\mathbf{A}+t \mathbf{B})^{-1} \mathbf{B}\right\}-N \operatorname{Tr}\{(\mathbf{A}+$ $\left.t \mathbf{B})^{-1} \mathbf{B}(\mathbf{A}+t \mathbf{B})^{-1} \mathbf{B}\right\} \leq 0$. Since

$$
\begin{aligned}
\operatorname{Tr}^{2}\left\{(\mathbf{A}+t \mathbf{B})^{-1} \mathbf{B}\right\} & =\operatorname{Tr}^{2}\left\{(\mathbf{A}+t \mathbf{B})^{-\frac{1}{2}} \mathbf{B}(\mathbf{A}+t \mathbf{B})^{-\frac{1}{2}}\right\} \\
& =\left(\sum_{i=1}^{r} \lambda_{i}\right)^{2}
\end{aligned}
$$

where $r$ is the rank of $(\mathbf{A}+t \mathbf{B})^{-1 / 2} \mathbf{B}(\mathbf{A}+t \mathbf{B})^{-1 / 2}$ and satisfies $r \leq N, \lambda_{i}$ is a eigenvalue of $(\mathbf{A}+t \mathbf{B})^{-1 / 2} \mathbf{B}(\mathbf{A}+$ $t \mathbf{B})^{-1 / 2}$, and

$$
\begin{aligned}
\operatorname{Tr}\{ & \left.(\mathbf{A}+t \mathbf{B})^{-1} \mathbf{B}(\mathbf{A}+t \mathbf{B})^{-1} \mathbf{B}\right\} \\
=\operatorname{Tr} & \left\{(\mathbf{A}+t \mathbf{B})^{-\frac{1}{2}} \mathbf{B}(\mathbf{A}+t \mathbf{B})^{-\frac{1}{2}}\right. \\
& \left.\times(\mathbf{A}+t \mathbf{B})^{-\frac{1}{2}} \mathbf{B}(\mathbf{A}+t \mathbf{B})^{-\frac{1}{2}}\right\} \\
= & \sum_{i=1}^{r} \lambda_{i}^{2}
\end{aligned}
$$

based on Cauchy-Schwarz inequality, it is not hard to get $\left(\sum_{i=1}^{r} \lambda_{i}\right)^{2} \leq r \sum_{i=1}^{r} \lambda_{i}^{2} \leq N \sum_{i=1}^{r} \lambda_{i}^{2}$. Thus, we have

$$
\begin{aligned}
\frac{d^{2} g(t)}{d t^{2}}=\frac{1}{N^{2}}|\mathbf{A}+t \mathbf{B}|^{\frac{1}{N}}\left[\operatorname{Tr}^{2}\left\{(\mathbf{A}+t \mathbf{B})^{-1} \mathbf{B}\right\}\right. \\
\left.-N \operatorname{Tr}\left\{(\mathbf{A}+t \mathbf{B})^{-1} \mathbf{B}(\mathbf{A}+t \mathbf{B})^{-1} \mathbf{B}\right\}\right] \leq 0
\end{aligned}
$$

which further indicates that $f_{1}\left(\mathbf{Q}_{1}\right)$ is concave. Next, we show that $g_{1}\left(\mathbf{Q}_{1}\right)=\left|\mathbf{I}_{N}+\mathbf{M}_{1} \mathbf{Q}_{1}\right|^{-1 / N}$ is convex. By introducing new variables $\mathbf{X}$ and $\mathbf{Y}$, we have

$$
\begin{aligned}
g_{1}(\alpha \mathbf{X}+(1-\alpha) \mathbf{Y}) & =\frac{1}{f_{1}(\alpha \mathbf{X}+(1-\alpha) \mathbf{Y})} \\
& \leq \frac{1}{\alpha f_{1}(\mathbf{X})+(1-\alpha) f_{1}(\mathbf{Y})} \\
& \leq \alpha \frac{1}{f_{1}(\mathbf{X})}+(1-\alpha) \frac{1}{f_{1}(\mathbf{Y})}
\end{aligned}
$$

where the first inequality is obtained by using the concavity of $f_{1}\left(\mathbf{Q}_{1}\right)$, and the second inequality is obtained by using the fact that $f(x)=x^{-1}, x \geq 0$ is a convex function. Thus, we conclude that $g_{1}\left(\mathbf{Q}_{1}\right)$ is convex. After extending this result to $f_{2}\left(\mathbf{Q}_{2}\right)$, we complete the proof of Lemma 1 .

\section{APPENDIX B \\ PROOF OF LEMMA 2}

To prove Lemma 2, we first give the following lemma:

Lemma B.1: The optimal objective value of (15) is lower bounded by

$$
\begin{aligned}
& q\left(\lambda_{1}, \lambda_{2}, \lambda_{3}\right)=\min _{\mathbf{Q}_{1}, \mathbf{Q}_{2} \succeq 0} \sum_{i=1}^{2}\left|\mathbf{I}_{N}+\mathbf{M}_{i} \mathbf{Q}_{i}\right|^{-\frac{1}{N}} \\
& \text { s.t. } \quad \operatorname{Tr}\left\{\left(\lambda_{1} \mathbf{I}+\lambda_{3} \mathbf{N}_{1}\right) \mathbf{Q}_{1}\right\}+\operatorname{Tr}\left\{\left(\lambda_{2} \mathbf{I}+\lambda_{3} \mathbf{N}_{2}\right) \mathbf{Q}_{2}\right\} \\
& \quad \leq \lambda_{1} P_{1}+\lambda_{2} P_{2}+\lambda_{3} P_{R}^{\prime}
\end{aligned}
$$


where $\lambda_{1}, \lambda_{2}$, and $\lambda_{3}$ are three nonnegative scalars. Moveover, this lower bound is tight and can be achieved by $\max _{\lambda_{1}, \lambda_{2}, \lambda_{3}} q\left(\lambda_{1}, \lambda_{2}, \lambda_{3}\right)$.

Since we have proven that the problem (15) is convex, the KKT conditions are sufficient and necessary for deriving the optimal solution. Then, the proof of Lemma B.1 is similar to the proof provided in [17, Props. 4 and 5]; thus, it is omitted for brevity. It is easy to verify that, in (41), the optimal solution must make the constraint active. We assume that the term $\operatorname{Tr}\left\{\left(\lambda_{1} \mathbf{I}+\lambda_{3} \mathbf{N}_{1}\right) \mathbf{Q}_{1}\right\}$ consumes power $t$ and that the term $\operatorname{Tr}\left\{\left(\lambda_{2} \mathbf{I}+\lambda_{3} \mathbf{N}_{2}\right) \mathbf{Q}_{2}\right\}$ consumes the remaining power of $\lambda_{1} P_{1}+\lambda_{2} P_{2}+\lambda_{3} P_{R}^{\prime}$. To derive the optimal structure of $\mathbf{Q}_{1}$, we only need to consider the following problem:

$$
\begin{array}{cl}
\max _{\mathbf{Q}_{1} \succeq 0} & \left|\mathbf{I}_{N}+\mathbf{M}_{1} \mathbf{Q}_{1}\right|^{\frac{1}{N}} \\
\text { s.t. } & \operatorname{Tr}\left\{\mathbf{Z}_{1} \mathbf{Q}_{1}\right\} \leq t .
\end{array}
$$

Since $\mathbf{Q}_{1}=\mathbf{F}_{1}^{\mathrm{lb}} \mathbf{F}_{1}^{\mathrm{lb} H}$, solving (42) is equivalent to solving the following problem:

$$
\begin{aligned}
& \underset{\mathbf{F}_{1}^{\mathrm{lb}}}{\max _{N}}\left|\mathbf{I}_{N}+\mathbf{F}_{1}^{\mathrm{lb} H} \mathbf{M}_{1} \mathbf{F}_{1}^{\mathrm{lb}}\right|^{\frac{1}{N}} \\
& \text { s.t. } \quad \operatorname{Tr}\left\{\mathbf{F}_{1}^{\mathrm{lb} H} \mathbf{Z}_{1} \mathbf{F}_{1}^{\mathrm{lb}}\right\} \leq t_{1} .
\end{aligned}
$$

For (43), without loss of generality, we assume that the optimal $\mathbf{F}_{1}^{\mathrm{lb}}$ makes the term $\mathbf{F}_{1}^{\mathrm{lb}}{ }^{H} \mathbf{M}_{1} \mathbf{F}_{1}^{\mathrm{lb}}$ diagonal. Otherwise, we can always multiply $\mathbf{F}_{1}^{\mathrm{lb}}$ with a unitary matrix (the eigenvector matrix of $\mathbf{F}_{1}^{\mathrm{lb} H} \mathbf{M}_{1} \mathbf{F}_{1}^{\mathrm{lb}}$ ) at the right side to make $\mathbf{F}_{1}^{\mathrm{lb} H} \mathbf{M}_{1} \mathbf{F}_{1}^{\mathrm{lb}}$ diagonal, and this new precoding matrix does not affect the value of the objective function and the power constraint. Thus, by assuming $\mathbf{F}_{1}^{\mathrm{lb}}{ }^{H} \mathbf{M}_{1} \mathbf{F}_{1}^{\mathrm{lb}}=\boldsymbol{\Sigma}_{1}$, where $\boldsymbol{\Sigma}_{1}$ is a diagonal matrix with the diagonal elements being arranged in the increasing order, we have $\mathbf{M}_{1}^{1 / 2} \mathbf{F}_{1}^{\mathrm{lb}}=\mathbf{U}_{1} \boldsymbol{\Sigma}_{1}$, where $\mathbf{U}_{1}$ is a unitary matrix, which will be determined later. Then, we have

$$
\mathbf{F}_{1}^{\mathrm{lb}}=\mathbf{M}_{1}^{-\frac{1}{2}} \mathbf{U}_{1} \boldsymbol{\Sigma}_{1}
$$

Substituting (44) into the power constraint (43) yields

$$
\begin{aligned}
\operatorname{Tr}\left\{\mathbf{F}_{1}^{\mathrm{lb}}{ }^{H} \mathbf{Z}_{1} \mathbf{F}_{1}^{\mathrm{lb}}\right\} & =\operatorname{Tr}\{\boldsymbol{\Sigma}_{1} \mathbf{U}_{1}^{H} \underbrace{\mathbf{M}_{1}^{-\frac{1}{2}} \mathbf{Z}_{1} \mathbf{M}_{1}^{-\frac{1}{2}}}_{\tilde{\mathbf{U}}_{1} \tilde{\boldsymbol{\Sigma}}_{1} \tilde{\mathbf{U}}_{1}^{H}} \mathbf{U}_{1} \boldsymbol{\Sigma}_{1}\} \\
& =\operatorname{Tr}\left\{\boldsymbol{\Sigma}_{1}^{2} \mathbf{U}_{1}^{H} \tilde{\mathbf{U}}_{1} \tilde{\boldsymbol{\Sigma}}_{1} \tilde{\mathbf{U}}_{1}^{H} \mathbf{U}_{1}\right\} \\
& \geq \operatorname{Tr}\left\{\boldsymbol{\Sigma}_{1}^{2} \tilde{\boldsymbol{\Sigma}}_{1}\right\}
\end{aligned}
$$

where the inequality is obtained by using the fact that $\operatorname{Tr}(\mathbf{A B}) \geq \operatorname{Tr}\left(\boldsymbol{\Lambda}_{A} \boldsymbol{\Lambda}_{B}\right)$, where $\boldsymbol{\Lambda}_{A}$ is a decreasing-ordered diagonal matrix with eigenvalues of $\mathbf{A}$ as its diagonal elements, and $\boldsymbol{\Lambda}_{B}$ is an increasing-ordered diagonal matrix with eigenvalues of $\mathbf{B}$ as its diagonal elements. Note that the equality in (45) holds when $\mathbf{U}_{1}=\tilde{\mathbf{U}}_{1}$. Thus, we conclude that the optimal structure of $\mathbf{F}_{1}^{\mathrm{lb}}$ is $\mathbf{M}_{1}^{-1 / 2} \tilde{\mathbf{U}}_{1} \boldsymbol{\Sigma}_{1}$, which further yields the optimal structure of $\mathbf{Q}_{1}$ as in (16). After extending this result to $\mathbf{Q}_{2}$, we complete the proof of Lemma 2 .

\section{REFERENCES}

[1] B. Rankov and A. Wittneben, "Spectral efficient protocols for half-duplex fading relay channels," IEEE J. Sel. Areas Commun., vol. 25, no. 2, pp. 379-389, Feb. 2007.

[2] S. Katti, H. Rahul, W. Hu, D. Katabi, M. Medard, and J. Crowcroft, "XORs in the air: Practical wireless network coding," IEEE/ACM Trans. Netw., vol. 16, no. 3, pp. 497-510, Jun. 2008.

[3] R. Zhang, Y.-C. Liang, C. C. Chai, and S. Cui, "Optimal beamforming for two-way multi-antenna relay channel with analogue network coding," IEEE J. Sel. Areas Commun., vol. 27, no. 5, pp. 699-712, Jun. 2009.

[4] G. Li, Y. Wang, and P. Zhang, "Optimal linear MMSE beamforming for two way multi-antenna relay systems," IEEE Commun. Lett., vol. 15, no. 5, pp. 533-535, May 2011.

[5] C. Li, L. Yang, and W.-P. Zhu, "Two-way MIMO relay precoder design with channel state information," IEEE Trans. Commun., vol. 58, no. 12, pp. 3358-3363, Dec. 2010.

[6] I. Hammerstrom, M. Kuhn, C. Esli, J. Zhao, A. Wittneben, and G. Bauch, "MIMO two-way relaying with transmit CSI at the relay," in Proc. IEEE SPAWC, 2007, pp. 1-5.

[7] R. Wang and M. Tao, "Joint source and relay precoding designs for MIMO two-way relay systems," in Proc. IEEE ICC, 2011, pp. 1-5.

[8] R. Wang and M. Tao, "Joint source and relay precoding designs for MIMO two-way relaying based on MSE criterion," IEEE Trans. Signal Process., vol. 60, no. 3, pp. 1352-1365, Mar. 2012.

[9] S. Xu and Y. Hua, "Optimal design of spatial source-and-relay matrices for a non-regenerative two-way MIMO relay system," IEEE Trans. Wireless Commun., vol. 10, no. 5, pp. 1645-1655, May 2011.

[10] J. Joung and A. H. Sayed, "Multiuser two-way amplify-and-forward relay processing and power control methods for beamforming systems," IEEE Trans. Signal Process., vol. 58, no. 3, pp. 1833-1846, Mar. 2010.

[11] Z. Ding, I. Krikidis, J. Thompson, and K. K. Leung, "Physical layer network coding and precoding for the two-way relay channel in cellular systems," IEEE Trans. Signal Process., vol. 59, no. 2, pp. 696-712, Feb. 2011.

[12] R. Wang and M. Tao, "Linear precoding designs for amplify-and-forward multiuser two-way relay systems," in Proc. IEEE GLOBECOM, 2011, pp. 1-6.

[13] F. Xu, T. N. Davidson, J.-K. Zhang, and K. M. Wong, "Design of block transceivers with decision feedback detection," IEEE Trans. Signal Process., vol. 54, no. 3, pp. 965-978, Mar. 2006.

[14] M. B. Shenouda and T. N. Davidson, "A framework for designing MIMO systems with decision feedback equalization or Tomlinson-Harashima precoding," IEEE J. Sel. Areas Commun., vol. 26, no. 2, pp. 401-411, Feb. 2008.

[15] Y. Rong, "Optimal linear non-regenerative multi-hop MIMO relays with MMSE-DFE receiver at the destination," IEEE Trans. Wireless Commun., vol. 9, no. 7, pp. 2268-2279, Jul. 2010.

[16] F.-S. Tseng and W.-R. Wu, "Nonlinear transceiver designs in MIMO amplify-and-forward relay systems," IEEE Trans. Veh. Technol., vol. 60, no. 2, pp. 528-538, Feb. 2011.

[17] L. Zhang, R. Zhang, Y.-C. Liang, Y. Xin, and H. V. Poor, "On Gaussian MIMO BC-MAC duality with multiple transmit covariance constraints," IEEE Trans. Inf. Theory, vol. 58, no. 4, pp. 2064-2078, Apr. 2012. [Online]. Available: http://arxiv.org/abs/0809.4101

[18] A. M. Stephen Boyd, "Subgradient methods," Stanford Univ., Stanford, CA, EE354b Course Notes, 2008.

[19] J.-K. Zhang, A. Kavcic, and K. M. Wong, "Equal-diagonal QR decomposition and its application to precoder design for successive-cancellation detection," IEEE Trans. Inf. Theory, vol. 51, no. 1, pp. 154-172, Jan. 2005

[20] M. Grant and S. Boyd, CVX: Matlab Software for Disciplined Convex Programming, Jul. 2010

[21] S. Boyd and L. Vandenberghe, Convex Optimization. Cambridge, U.K.: Cambridge Univ. Press, 2004.

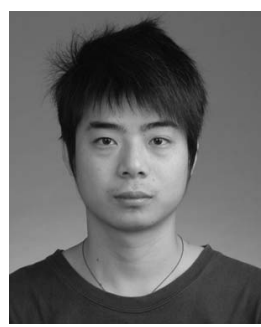

Rui Wang received the B.S. degree in electronic engineering from Anhui Normal University, Wuhu, China, in 2006 and the M.S. degree in electronic engineering from Shanghai University, Shanghai, China, in 2009. He is currently working toward the Ph.D. degree with the Institute of Wireless Communication Technology, Shanghai Jiao Tong University.

His research interests include digital image processing, cognitive radio, and signal processing for wireless cooperative communication. 


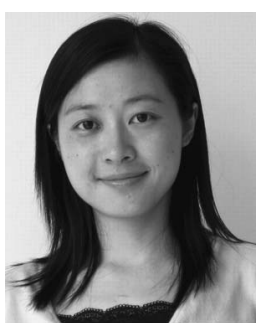

Meixia Tao (S'00-M'04-SM'10) received the B.S. degree in electronic engineering from Fudan University, Shanghai, China, in 1999 and the Ph.D. degree in electrical and electronic engineering from Hong Kong University of Science and Technology, Kowloon, Hong Kong, in 2003.

She is currently an Associate Professor with the Department of Electronic Engineering, Shanghai Jiao Tong University. From August 2003 to August 2004, she was a member of Professional Staff with Hong Kong Applied Science and Technology Research Institute Co. Ltd. From August 2004 to December 2007, she was with the Department of Electrical and Computer Engineering, National University of Singapore, Singapore, as an Assistant Professor. Her current research interests include cooperative transmission, physical-layer network coding, resource allocation of orthogonal frequency-division multiplexing networks, and multipleinput-multiple-output techniques.

Dr. Tao is an Associate Editor for the IEEE COMMUNICATIONS LETTERS and an Editor for the IEEE Wireless COMMUNiCATIONS LETTERS. She was on the Editorial Board of the IEEE TRANSACTIONS ON WIRELESS Communications from 2007 to 2011. She served as Track/Symposium CoChair for the 2012 International Symposium on Wireless Personal Multimedia Communications; the 2009 Asia-Pacific Conference on Communications; the 2009 International Conference on Communications and Networking in China; the 2007 IEEE International Conference on Computer Communications and Networks; and the 2007 IEEE International Conference on Communications, Circuits, and Systems. She has also served as Technical Program Committee member for various conferences, including the IEEE International Conference on Computer Communications, the IEEE Global Communications Conference, the IEEE International Conference on Communications, the IEEE Wireless Communications and Networking Conference, and the IEEE Vehicular Technology Conference. She received the IEEE Communications Society AsiaPacific Outstanding Young Researcher Award in 2009.

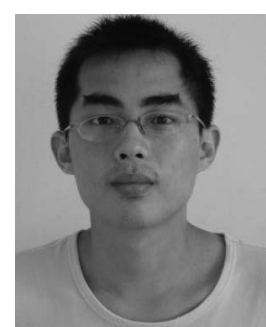

Zhengzheng Xiang received the B.S. degree in electronic engineering in 2010 from Shanghai Jiao Tong University, Shanghai, China, where he is currently working toward the Ph.D. degree with the Institute of Wireless Communication Technology.

His research interests include interference management in wireless networks, wireless relay technologies, and advanced signal processing for wireless cooperative communication. 